\title{
First-principles investigation of dynamical properties of molecular devices under a steplike pulse
}

\author{
Yanxia Xing, ${ }^{1,2}$ Bin Wang, ${ }^{1}$ and Jian Wang ${ }^{1, *}$ \\ ${ }^{1}$ Department of Physics and the Center of Theoretical and Computational Physics, The University of Hong Kong, Pokfulam Road, \\ Hong Kong, China \\ ${ }^{2}$ Department of Physics, Beijing Institute of Technology, Beijing 100081, China \\ (Received 18 June 2010; revised manuscript received 9 September 2010; published 10 November 2010)
}

\begin{abstract}
We report a computationally tractable approach to first-principles investigation of time-dependent current of molecular devices under a steplike pulse. For molecular devices, all the resonant states below Fermi level contribute to the time-dependent current. Hence calculation beyond wideband limit must be carried out for a quantitative analysis of transient dynamics of molecules devices. Based on the exact nonequilibrium Green'sfunction (NEGF) formalism of calculating the transient current [J. Maciejko, J. Wang, and H. Guo, Phys. Rev. B 74, 085324 (2006)], we develop two approximate schemes going beyond the wideband limit, they are all suitable for first-principles calculation using the NEGF combined with density-functional theory. Benchmark test has been done by comparing with the exact solution of a single level quantum dot system. Good agreement has been reached for two approximate schemes. As an application, we calculate the transient current using the first approximated formula with opposite voltage $V_{L}(t)=-V_{R}(t)$ in two molecular structures: Al- $\mathrm{C}_{5}-\mathrm{Al}$ and $\mathrm{Al}-\mathrm{C}_{60}-\mathrm{Al}$. As illustrated in these examples, our formalism can be easily implemented for real molecular devices. Importantly, our new formula has captured the essential physics of dynamical properties of molecular devices and gives the correct steady state current at $t=0$ and $t \rightarrow \infty$.
\end{abstract}

DOI: 10.1103/PhysRevB.82.205112

PACS number(s): $71.15 . \mathrm{Mb}$

\section{INTRODUCTION}

With the rapid progress in molecular electronics, ${ }^{1}$ quantum transport in molecular device has received increasing attention. In particular, the dynamic response of molecular devices to external parameters, ${ }^{2-8}$ in which the external timedependent fields or internal parametric pump potentials drive the electrons to tunnel through the molecular device, is one of the most important issues in molecular electronics. The simplest molecular device structure is the two-probe leaddevice-lead configuration, where "device" is the molecular device connected to the external probes by the "leads." In such a device, all the atomic details of the device material can be treated using density-functional theory (DFT) and the nonequilibrium physics can be taken into account using nonequilibrium Green's function (NEGF). Up to now, from an atom point of view, one of the most popular theoretical approaches used to study the quantum transport properties of molecular device is Keldysh nonequilibrium Green's functions coupled with density-functional theory (NEGF-DFT). ${ }^{9}$ Using this approach, the steady state quantum transport properties in molecular devices have been widely studied.

For time-dependent response of molecular devices, there have been many different theoretical approaches, such as evolution of time-dependent Schrodinger equation, ${ }^{10}$ time development operator approach, ${ }^{11}$ and the NEGF technique. ${ }^{12}$ These approaches are convenient to deal with dynamic response of time-dependent external field that is sinusoidal (e.g., microwave radiation). Under such an external field an electron can tunnel through the system by emitting or absorbing photons, giving rise to the photon-assisted tunneling (PAT). Concerning the steady state ac response to harmonic external field, the Floquet approach is convenient. ${ }^{13}$ For the transient transport, however, the pulselike ac signal is the optimal driven force since they can pro- vide a less ambiguous measure of time scales. ${ }^{14}$ In this case, besides PAT, one of the most interesting questions to ask is how fast a device can turn on or turn off a current. With the development of molecular electronics, providing a particular viable switching device has become a key technical issue. Concerning the transient dynamics, different approaches such as path-integral techniques, ${ }^{15}$ the solution of Wigner distribution function, ${ }^{16}$ the time-dependent numerical renormalization group, ${ }^{17}$ time-dependent DFT, ${ }^{2,18}$ and Keldish Green's function ${ }^{3,5,19}$ have also been developed and applied to different systems. Up to now, most of these approaches can only be implemented in simple systems such as quantum $\operatorname{dots}^{5,19}$ or one-dimension tight-binding chains. ${ }^{2}$ Numerical calculation of transient current for molecular devices is very difficult at present stage due to the huge computational cost. This is because if we calculate the current as a function of time $t$, the amount of calculation scales as $t^{3}$ if the timeevolution method is used. This scaling can be reduced to a linear scaling in $t$ if the wideband limit is used. ${ }^{20}$ As we have demonstrated, ${ }^{21}$ the wideband limit is not a good approximation for molecular devices. If one uses the exact solution from $\mathrm{NEGF}^{5}$ one can calculate the transient current at a particular time. However, the calculation involves a triple integration over energy which is extremely time consuming. Clearly an approximate scheme that is suitable for numerical calculation of transient properties for real molecular devices while still captured essential physics is needed.

It is the purpose of this paper to provide such a practical scheme. To study transient dynamics, in this paper, we consider a system that consists of a scattering region coupled to two leads with the external time-dependent pulse bias potential $V_{\alpha}(t)=\theta( \pm t) V_{\alpha}$. For this case, the time-dependent current for a steplike pulse has been derived exactly without using the wide-band limit by Maciejko et al. ${ }^{7}$ Since the general expression for the current involves triple integrations, it is extremely difficult to perform them in a real systems such 
as molecules devices. So, approximation has to be made. The simplest approximation is the so called wide-band approximation where self-energies $\Sigma^{r, a}$ are assumed to be constants independent of energy. ${ }^{22}$ Unfortunately, this approximation cannot give the correct result since in general there are several resonant levels that significantly contribute to the transient current in molecules devices. To go beyond the wideband limit, we propose an approximate scheme of calculating the transient current that is suitable for numerical calculation of real molecules devices. ${ }^{23}$ Our scheme is an approximation of the exact solution of Maciejko et al. ${ }^{5}$ It is very fast computationally and gives the correct limits at $t=0$ and $t=\infty$. Since the exact solution of transient current is available for a single level quantum dot system, we have compared our result with the exact solution on the quantum dot system to test our approximate schemes. Good agreement is obtained. Therefore, our approximated scheme maintains essential physics of transient dynamics. Using our scheme, we calculate the transient current for the upward pulse (turn-on) and downward pulse (turn-off) in two molecular structures: $\mathrm{Al}-\mathrm{C}_{5}-\mathrm{Al}$ and $\mathrm{Al}-\mathrm{C}_{60}-\mathrm{Al}$. We find that different from the single level quantum dot system, upon switching on the current oscillates rapidly in the first a few or tens femtoseconds with several characteristic time scales. Furthermore, due to the resonant states in molecular devices, transient currents have a much longer decay time $\tau$, especially for the molecule device having a complex electronic structure such as Al- $\mathrm{C}_{60}-\mathrm{Al}$.

The rest of paper is organized as follows: In Sec. II, starting from the typical molecular device Hamiltonian which is expressed in an nonorthogonal basis, we shall derive a general dc and ac current expressions for a nonorthogonal basis set. It is found that for dc bias, the expressions of current for orthogonal and nonorthogonal basis sets are the same. For ac current, however, the expressions are different as will be demonstrated in Sec. II. The reason that we study the difference between orthogonal and nonorthogonal basis sets is the following. For the NEGF formalism, it is assumed that the basis set is orthogonal. It turns out that for ac transport, the current expression becomes extremely complicated if nonorthogonal basis is used. For DFT calculation, however, most people work in molecular orbitals that are nonorthogonal. Our results show that we must orthogonalizing the nonorthogonal molecular Hamiltonian, so that the present approach in Ref. 5 can be used. In Sec. III, based on the exact solution of Maciejko et al., we derive two approximate expressions for transient current with different levels of approximation. They are all suitable for numerical calculation for real molecular devices. In addition, the initial current and its asymptotic long time limit are shown to be correct. In $\mathrm{Sec}$ IV, in order to appreciate our approximate formulas, we compare our result with the exact result obtained in Ref. 5 for a single-level quantum dot connected to external leads with a Lorentzian linewidth. In Sec. V, we apply our formalism to several molecular devices. Finally, a conclusion is presented in Sec. VI. Two appendices are given at the end of the paper. In Appendix A, we give a detailed derivation of orthogonalization relation for a nonorthogonal basis. This relation is used to derive the effective Green's function which is the key to approximate exact current expression of Macie- jko et al. In Appendix B, we show how to orthogonalize an nonorthogonal Hamiltonian so that the general ac current for real molecules device can be derived.

\section{GENERAL ac CURRENT}

\section{A. Hamiltonian}

The transport properties of a molecular device can be described by the following general Hamiltonian:

$$
H=H_{c}+H_{T}+\sum_{\alpha=L, R} H_{\alpha},
$$

where $H_{L}$ and $H_{R}$ describe the left and right macroscopic reservoir, respectively; $H_{c}$ is Hamiltonian of the central molecular device; $H_{T}$ couples the reservoirs to the molecular device. For a particular basis set, the above Hamiltonian can be written in the following matrix form:

$$
\begin{gathered}
H_{\alpha}=\sum_{\mu_{\alpha} \nu_{\alpha}} c_{\mu_{\alpha}}^{\dagger}\left[\mathbf{H}_{\mu_{\alpha} \nu_{\alpha}}^{0}+e V_{\alpha}(t) \delta_{\mu_{\alpha} \nu_{\alpha}}\right] c_{\nu_{\alpha}}, \\
H_{c}=\sum_{\mu_{c} \nu_{c}} d_{\mu_{c}}^{\dagger}\left[\mathbf{H}_{\mu_{c} \nu_{c}}^{0}+\mathbf{U}_{\mu_{c} \nu_{c}}(t)\right] d_{\nu_{c}}, \\
H_{T}=\sum_{\nu_{\alpha}, \nu_{c}} c_{\nu_{\alpha}}^{\dagger} \mathbf{T}_{\nu_{\alpha} \nu_{c}}^{0} d_{\nu_{c}}+\text { h.c. },
\end{gathered}
$$

where $e$ is the electron charge, $c_{\nu_{\alpha}}\left(c_{\nu_{\alpha}^{\dagger}}^{\dagger}\right)$ and $d_{\nu_{c}}\left(d_{\nu_{c}}^{\dagger}\right)$ are Fermionic annihilation (creation) operators at the state $\nu$ in the lead- $\alpha$ and the state $\nu$ in central molecular device. $\nu_{\alpha}, \nu_{c}$ are the indices of the given basis set. The Hamiltonian of lead- $\alpha$ are divided into two parts: the time independent part $\mathbf{H}_{\alpha}^{0}$ and time-dependent part due to external bias $V_{\alpha}(t)$ on the lead- $\alpha$. Here we consider two kinds of steplike bias: upward pulse (turn-on case) $V_{\alpha}^{U}(t)$ and downward pulse (turn-off case) $V_{\alpha}^{D}(t)$, where

$$
V_{\alpha}^{D}(t)=\left\{\begin{array}{ll}
V_{\alpha}, & t<0 \\
0, & t>0
\end{array}, \quad V_{\alpha}^{U}(t)= \begin{cases}0, & t<0 \\
V_{\alpha}, & t>0\end{cases}\right.
$$

In the adiabatic approximation it is assumed that the single particle energies acquire a rigid time-dependent shift as $\mathbf{H}_{\alpha}^{0}$ $+\mathbf{I} V_{\alpha}(t)$. The energy shift in the leads is assumed to be uniform throughout. This assumption is reasonable since the pulse rising time is slower than the usual metallic plasma oscillation time, which ensures that the external electric field is effectively screened. ${ }^{24}$

Since Green's function $\mathbf{G}^{r}\left(t, t^{\prime}\right)$ is obtained by solving Dyson equation from the known history, it is better to set time-dependent external bias $V_{\alpha}(t>0)=0$ so that the uncertainty of future can be eliminated. ${ }^{5}$ From Eq. (3), this is satisfied only in the downward case. In the following, we will discuss how to eliminate this uncertainty for the upward pulse. To use the Dyson equation, we will separate the Hamiltonian into two pieces: the unperturbed Hamiltonian that can be exactly resolved and the interacting term which contributes to the self-energy in Dyson equations. For the downward pulse, we define the nonbiased open system as the unperturbed system. It is described by the Hamiltonian $\mathbf{H}^{0}$ 
$=\mathbf{H}_{\alpha}^{0}+\mathbf{H}_{c}^{0}+\mathbf{H}_{T}^{0}$. For the upward pulse, however, the situation is different, in which we will set the dc biased open system $\mathbf{H}^{V}=\left[\mathbf{H}_{\alpha}^{0}+V_{\alpha} \mathbf{I}\right]+\left[\mathbf{H}_{c}^{0}+\mathbf{U}^{V}\right]+\mathbf{H}_{T}^{V}$ as the unperturbed Hamiltonian and set $\widetilde{V}_{\alpha}^{U}(t)=V_{\alpha}^{U}(t)-V_{\alpha}$ as the new time-dependent part. Here $\mathbf{H}_{T}^{V}$ denotes the coupling between scattering region and biased leads and $\mathbf{U}^{V}$ is the induced coulomb potential due to the external bias. Now, the time-dependent bias $\widetilde{V}^{U}$ satisfies $\widetilde{V}^{U}(t>0)=0$, and the uncertainty of the future in the upward case is eliminated. Then, for the downward case, we have $\widetilde{V}_{\alpha}^{D}(t)=V_{\alpha}^{D}(t)$ and $\mathbf{H}^{e r}=\mathbf{H}^{0}$ while for the upward case we have $\widetilde{V}_{\alpha}^{U}(t)=V_{\alpha}^{U}(t)-V_{\alpha}$ and $\mathbf{H}^{e x}=\mathbf{H}^{V}$. From now on we will use superscript "ex" to denote the unperturbed system that is exactly resolvable.

When the system is biased, the incoming electron will polarize the system. The induced Coulomb potential in the central scattering region consists of two parts: dc and ac parts. The dc part can be put into the exactly resolvable Hamiltonian $\mathbf{H}^{e x}$. The induced time-dependent coulomb potential $\mathbf{U}(t)$ due to the external bias $\widetilde{V}_{\alpha}(t)$ is included as part of the nonequilibrium Hamiltonian. Because the electric field is not screened in the small scattering region where the potential drop occurs, the coulomb potential landscape $\mathbf{U}(\mathbf{t})$ in the central region is not uniform, which is different from the semi-infinite leads. Note that it is rather difficult to treat the time-dependent coulomb potential and no close formed solution exists if one does not assume wide band limit. In the small bias limit, we can expand the time-dependent coulomb potential to linear order in bias $\mathbf{U}(t)=e \Sigma_{\alpha} \mathbf{u}_{\alpha} \widetilde{V}_{\alpha}(t)$ so that the analytic expression for current can be obtained. Here $u_{\alpha}$ is the characteristic potential. ${ }^{26}$ From the gauge invariance, ${ }^{25}$ $\Sigma_{\alpha} \mathbf{u}_{\alpha}=\mathbf{I}$, and $\mathbf{u}_{\alpha}$ is determined from a Poisson-type equation. ${ }^{27}$ In this paper, we consider the symmetric coupling so that for the external bias $\widetilde{V}_{L}(t)=-\widetilde{V}_{R}(t)$ it is a good approximation to assume that the time-dependent coulomb potential $U(t)$ is roughly zero in the molecular device regime.

In the following, we will derive an exact solution of transient current using a nonorthogonal basis set. ${ }^{28}$ To facilitate the derivation, we take a unitary transformation $\hat{O}(t)$ to Hamiltonian (2) with

$$
\hat{O}(t)=\exp \left\{i e \sum_{\nu_{\alpha}} \int_{0}^{t} d \tau\left[\tilde{V}_{\alpha}(\tau) c_{\nu_{\alpha}}^{\dagger} c_{\nu_{\alpha}}\right]\right\},
$$

where $\widetilde{V}_{\alpha}(\tau)=\theta(-\tau) V_{\alpha}$ for the downward pulse and $\widetilde{V}_{\alpha}(\tau)=$ $-\theta(-\tau) V_{\alpha}$ for the upward pulse. Note that the time $t$ in $\hat{O}(t)$ can be negative or positive, and $\hat{O}(t)=1$ only when $t>0$. The new Hamiltonian $\mathcal{H}=\hat{O} H \hat{O}^{\dagger}(t)+i\left[\frac{\partial}{\partial t} \hat{O}(t)\right] \hat{O}^{\dagger}(t)$, in which $\mathcal{H}_{\alpha}$ and $\mathcal{H}_{T}$ are different from original ones

$$
\begin{gathered}
\mathcal{H}_{\alpha}=\sum_{\mu_{\alpha} \nu_{\alpha}} \bar{c}_{\mu_{\alpha}}^{\dagger} \mathbf{H}_{\mu_{\alpha} \nu_{\alpha}}^{0} \bar{c}_{\nu_{\alpha}}, \\
\mathcal{H}_{T}=\sum_{\nu_{\alpha} \nu_{c}} \bar{c}_{\nu_{\alpha}}^{\dagger} \mathbf{T}_{\nu_{\alpha} \nu_{c}}(t) d_{\nu_{c}}+\text { h.c. }
\end{gathered}
$$

where

$$
\begin{gathered}
\bar{c}_{\nu_{\alpha}}=c_{\nu_{\alpha}} \exp \left[i e \sum_{\mu_{\alpha}} \int_{0}^{t} d \tau \tilde{V}_{\alpha}(\tau) c_{\mu_{\alpha}}^{\dagger} c_{\mu_{\alpha}}\right], \\
\mathbf{T}_{\nu_{\alpha} \nu_{c}}(t)=\mathbf{T}_{\nu_{\alpha} \nu_{c}}^{0} \mathfrak{W}_{\alpha}(t) \\
\mathfrak{W}_{\alpha}(t)=\exp \left[i e \int_{0}^{t} \tilde{V}_{\alpha}(\tau) d \tau\right] .
\end{gathered}
$$

For the original Hamiltonian with nonorthogonal basis, the overlap between nonorthogonal basis is expressed as the matrix form $\mathbf{S}_{\mu \nu}^{0}=\langle\mu \mid \nu\rangle$. After the unitary transform, annihilation (creation) operators $c_{\alpha}\left(c_{\alpha}^{\dagger}\right)$ and consequently the orbital basis $\mu_{\alpha}$ in the leads are changed, then overlap matrices between the leads and the scattering region become

$$
\begin{aligned}
& \mathbf{S}_{\nu_{\alpha} \nu_{c}}(t)=\mathbf{S}_{\nu_{\alpha} \nu_{c}}^{0} \mathfrak{W}_{\alpha}(t), \\
& \mathbf{S}_{\nu_{c} \nu_{\alpha}}(t)=\mathfrak{W}_{\alpha}^{\dagger}(t) \mathbf{S}_{\nu_{c} \nu_{\alpha}}^{0} .
\end{aligned}
$$

In the following, we will use the transformed Hamiltonian [Eqs. (4) and (5), in which $\bar{c}_{\nu_{\alpha}}, d_{\nu_{c}}$ are used] to derive the time-dependent current expression.

\section{B. Current}

The current operator from a particular lead- $\alpha$ to the molecular junction can be calculated from the evolution of the number operator of the electron in the semi-infinite lead- $\alpha$. Assuming there is no direct coupling between the left and right leads, the current operator can be expressed as ${ }^{29}$

$$
\begin{aligned}
\hat{J}_{\alpha}(t)= & -e \sum_{\nu_{\alpha}} \frac{d}{d t} \hat{N}_{\nu_{\alpha}}(t)=-e \sum_{\nu_{\alpha}}\left\{\bar{c}_{\nu_{\alpha}}^{\dagger}(t) \frac{d}{d t} \bar{c}_{\nu_{\alpha}}(t)\right. \\
& \left.+\left[\frac{d}{d t} \bar{c}_{\nu_{\alpha}}^{\dagger}(t)\right] \bar{c}_{\nu_{\alpha}}(t)\right\} \\
= & e \sum_{\nu_{\alpha} \nu_{c}} \bar{c}_{\nu_{\alpha}}^{\dagger}(t)\left[i \mathbf{T}_{\nu_{\alpha} \nu_{c}}(t)+\mathbf{S}_{\nu_{\alpha} \nu_{c}}(t) \frac{d}{d t}\right] d_{\nu_{c}}(t)+\text { H.c. },
\end{aligned}
$$

where "H.c." denotes the Hermitian conjugate. The current is obtained by taking average over the nonequilibrium quantum state " $(\cdots\rangle$, ,

$$
\begin{aligned}
J_{\alpha}(t)= & e \sum_{\nu_{\alpha} \nu_{c}}\left\{\mathbf{G}_{\nu_{c} \nu_{\alpha}}^{<}\left(t, t^{\prime}\right)\left[\mathbf{T}_{\nu_{\alpha}, \nu_{c}}\left(t^{\prime}\right)-\mathbf{S}_{\nu_{\alpha}, \nu_{c}}\left(t^{\prime}\right) i \frac{\partial}{\partial t}\right]\right. \\
& \left.-\left[\mathbf{T}_{\nu_{c}, \nu_{\alpha}}\left(t^{\prime}\right)-\mathbf{S}_{\nu_{c}, \nu_{\alpha}}\left(t^{\prime}\right) i \frac{\partial}{\partial t}\right] \mathbf{G}_{\nu_{\alpha} \nu_{c}}^{<}\left(t^{\prime}, t\right)\right\}_{t=t^{\prime}},
\end{aligned}
$$

where “ $\frac{\partial}{\partial t}$ " and “ $\frac{\partial}{\partial t}$ " denotes the left and right derivation, respectively, and

$$
\mathbf{G}_{\nu_{c}, \nu_{\alpha}}^{<}\left(t, t^{\prime}\right)=i\left\langle\bar{c}_{\nu_{\alpha}}^{\dagger}\left(t^{\prime}\right) d_{\nu_{c}}(t)\right\rangle, \quad \mathbf{G}_{\nu_{\alpha}, \nu_{c}}^{<}\left(t^{\prime}, t\right)=i\left\langle d_{\nu_{c}}^{\dagger}(t) \bar{c}_{\nu_{\alpha}}\left(t^{\prime}\right)\right\rangle .
$$

Using the Keldysh equation and the theorem of analytic continuation, we have 


$$
\begin{aligned}
\mathbf{G}_{c \alpha}^{<}\left(t, t^{\prime}\right)= & \int d t_{1}\left[\mathbf{G}_{c c}^{r}\left(t, t_{1}\right) \mathbf{B}_{c \alpha}\left(t_{1}\right) \mathbf{g}_{\alpha \alpha}^{<}\left(t_{1}, t^{\prime}\right)\right. \\
& \left.+\mathbf{G}_{c c}^{<}\left(t, t_{1}\right) \mathbf{B}_{c \alpha}\left(t_{1}\right) \mathbf{g}_{\alpha \alpha}^{a}\left(t_{1}, t^{\prime}\right)\right],
\end{aligned}
$$

where

$$
\mathbf{B}_{c \alpha}\left(t_{1}\right)=\mathbf{T}_{c \alpha}\left(t_{1}\right)-\mathbf{S}_{c \alpha}\left(t_{1}\right) i \frac{\partial}{\partial t} \text {. }
$$

For simplicity, we have dropped the subscript $\mu$, and keep only the symbol $c$ and $\alpha$ to indicate the central scattering region and lead- $\alpha$, respectively. In the above expression and in the following, the summation convention on repeated subindices is assumed. Substituting Eq. (9) into Eq. (8), we have the general expression for the current

$$
\begin{aligned}
J_{\alpha}(t)= & -2 e \operatorname{Re} \int d t_{1} \operatorname{Tr}\left[\mathbf{G}_{c c}^{r}\left(t, t_{1}\right) \mathbf{B}_{c \alpha}\left(t_{1}\right) \mathbf{g}_{\alpha \alpha}^{<}\left(t_{1}, t^{\prime}\right) \mathbf{B}_{\alpha c}\left(t^{\prime}\right)\right. \\
& \left.-\mathbf{G}_{c c}^{<}\left(t, t_{1}\right) \mathbf{B}_{c \alpha}\left(t_{1}\right) \mathbf{g}_{\alpha \alpha}^{a}\left(t_{1}, t^{\prime}\right) \mathbf{B}_{\alpha c}\left(t^{\prime}\right)\right]_{t=t^{\prime}} .
\end{aligned}
$$

When the system reaches a stationary state, $V_{\alpha}(t)=V_{\alpha}$ becomes time independent, from definition Eqs. (5), (6), and (10), we can find

$$
\mathbf{B}_{c \alpha}\left(t_{1}\right) X \mathbf{B}_{\alpha c}(t)=e^{-i e V_{\alpha}\left(t_{1}-t\right)} \mathbf{B}_{c \alpha}^{0} X \mathbf{B}_{\alpha c}^{0}
$$

with $\mathbf{B}_{c \alpha / \alpha c}^{0}=\mathbf{T}_{c \alpha / \alpha c}^{0}-i \frac{\partial}{\partial t} \mathbf{S}_{c \alpha / \alpha c}^{0}$, where " 0 " denotes the zero bias system. In addition, all the propagators $\mathbf{G}$ and $\mathbf{g}$ depend only on the time difference $t_{1}-t$. Taking the Fourier transformation, from Eq. (8) or Eq. (11), we can easily obtain dc current expressed in the energy representation

$J_{\alpha}=\int d \epsilon \mathcal{J}_{\alpha}(\epsilon)=\operatorname{Re} 2 \mathrm{e} \int \mathrm{d} \epsilon \operatorname{Tr}\left[\mathbf{G}^{\mathrm{r}}(\epsilon) \mathbf{\Sigma}_{\alpha}^{<}(\epsilon)+\mathbf{G}^{<}(\epsilon) \boldsymbol{\Sigma}_{\alpha}^{\mathrm{a}}(\boldsymbol{\epsilon})\right]$,

where $\mathbf{G}$ and $\mathbf{\Sigma}$ are the Green's function and the self-energy. They have the same matrix dimension as that of the Hamiltonian $\mathbf{H}_{c}$. The Green's function $\mathbf{G}^{r / a}$ and self-energy $\mathbf{\Sigma}^{r / a}$ is defined as

$$
\begin{gathered}
\mathbf{G}^{r / a}(\boldsymbol{\epsilon})=\left[\boldsymbol{\epsilon} \mathbf{I}-\mathbf{H}_{c}-\boldsymbol{\Sigma}^{r / a}(\boldsymbol{\epsilon})\right]^{-1}, \\
\boldsymbol{\Sigma}_{\alpha}^{\gamma}(\boldsymbol{\epsilon})=\left[\mathbf{T}_{c \alpha}^{0}-\boldsymbol{\epsilon}^{\alpha} \mathbf{S}_{c \alpha}^{0}\right] \mathbf{g}_{\alpha \alpha}^{\gamma}\left(\boldsymbol{\epsilon}^{\alpha}\right)\left[\mathbf{T}_{\alpha c}^{0}-\boldsymbol{\epsilon}^{\alpha} \mathbf{S}_{\alpha c}^{0}\right]
\end{gathered}
$$

where $\epsilon^{\alpha}=\epsilon-e V_{\alpha}$, I is the unitary matrix with same dimension as $\mathbf{H}_{c}, \gamma=r, a,<$, and

$$
\begin{aligned}
\mathbf{g}_{\alpha \alpha}^{r / a}(\boldsymbol{\epsilon})= & \left\{\left[\left(\boldsymbol{\epsilon} \pm i 0^{+}\right) \mathbf{S}_{\alpha \alpha}^{0}-\mathbf{H}_{\alpha \alpha}^{0}\right]^{-1}\right\}_{\nu_{\alpha} \in \operatorname{sur}, \mu_{\alpha} \in \text { sur }}, \\
& \mathbf{g}_{\alpha \alpha}^{<}(\boldsymbol{\epsilon})=f(\epsilon)\left[\mathbf{g}_{\alpha \alpha}^{a}(\boldsymbol{\epsilon})-\mathbf{g}_{\alpha \alpha}^{r}(\boldsymbol{\epsilon})\right]
\end{aligned}
$$

is the surface Green's function of the semi-infinite periodic lead which can be calculated numerically using a transfer matrix method. ${ }^{30}$ Here, $f(\epsilon)$ is the Fermi distribution. Equation (12) shows that the dc current expressions are the same for both orthogonal and nonorthogonal basis sets.

When the time-dependent field $V_{\alpha}(t)$ is present, however, the current expressed in energy representation will be very complicated for nonorthogonal basis due to the term $\mathbf{S}\left(t^{\prime}\right) i \frac{\partial}{\partial t}$ in Eq. (8) since $\mathbf{B}\left(t_{1}\right) X \mathbf{B}(t)$ cannot be expressed as a function of time difference $t_{1}-t$. One thing is clear, the transient current expressions are different for orthogonal and nonorthogonal basis sets. Instead of deriving a complicated transient current expression using a nonorthogonal basis set, we will eliminate $\mathbf{S}_{c \alpha / \alpha c}\left(t^{\prime}\right) i \frac{\partial}{\partial t}$ in Eq. (8) and work on an orthogonal basis set. In Appendix B, from the overlap matrix $\mathbf{S}$, we derive the orthogonal basis set and new Hamiltonian $\widetilde{H}$ expressed in this orthogonal basis. With the new orthogonal Hamiltonian, the overlap matrix $\mathbf{S}_{c \alpha / \alpha c}\left(t^{\prime}\right)$ will be eliminated since the overlap matrix of orthogonal basis $\mathbf{S}^{\text {orth }}=\mathbf{I}$. Then, replacing Hamiltonian $\mathbf{H}$ in Eq. (2) with $\tilde{\mathbf{H}}$ and go through the derivation leading to Eqs. (2)-(11) again, we arrive at a new ac current expression

$$
\begin{aligned}
J_{\alpha}(t)= & 2 e \operatorname{Re} \int d t_{1} \operatorname{Tr}\left\{\mathbf{G}_{c c}^{r}\left(t, t_{1}\right)\left[\mathbf{T}_{c \alpha}\left(t_{1}\right) \mathbf{g}_{\alpha \alpha}^{<, e x}\left(t_{1}-t\right) \mathbf{T}_{\alpha c}(t)\right]\right\} \\
& +2 e \operatorname{Re} \int d t_{1} \operatorname{Tr}\left\{\mathbf{G}_{c c}^{<}\left(t, t_{1}\right)\left[\mathbf{T}_{c \alpha}\left(t_{1}\right) \mathbf{g}_{\alpha \alpha}^{a, e x}\left(t_{1}-t\right) \mathbf{T}_{\alpha c}(t)\right]\right\}
\end{aligned}
$$

Defining the self-energy on the orthogonal basis

$$
\boldsymbol{\Sigma}_{\alpha}^{\gamma=r, a,<}\left(t, t^{\prime}\right)=\mathbf{T}_{c \alpha}(t) \mathbf{g}_{\alpha \alpha}^{\gamma, e x}\left(t-t^{\prime}\right) \mathbf{T}_{\alpha c}\left(t^{\prime}\right),
$$

where $\mathbf{g}_{\alpha \alpha}^{\gamma, e x}\left(t-t^{\prime}\right)=\int \frac{d \epsilon}{2 \pi} e^{-i \epsilon\left(t-t^{\prime}\right)} \mathbf{g}_{\alpha \alpha}^{\gamma, e x}(\epsilon)$ is the surface Green's function of semi-infinite lead- $\alpha$ in the unperturbed state as defined in the Sec. II A. For the downward pulse we have set the unperturbed system as the open system at zero bias, in which $\mathbf{g}_{\alpha \alpha}^{\gamma, e x}(\epsilon)=\left[\epsilon-H_{\alpha}^{0}+i 0^{+}\right]_{\alpha \in \text { sur. }}^{-1}$. For the upward pulse, the unperturbed system means $V_{\alpha}$ biased open system, in which $\mathbf{g}_{\alpha \alpha}^{\gamma, e q}(\epsilon)=\left[\epsilon-e V_{\alpha}-H_{\alpha}^{0}+i 0^{+}\right]_{\alpha \in \text { sur. }}^{-1}$ From Eqs. (15) and (16), we have the general current formula

$$
J_{\alpha}(t)=2 e \operatorname{Re} \int d t_{1} \operatorname{Tr}\left[\mathbf{G}^{r}\left(t, t_{1}\right) \Sigma_{\alpha}^{<}\left(t_{1}, t\right)+\mathbf{G}^{<}\left(t, t_{1}\right) \Sigma_{\alpha}^{a}\left(t_{1}, t\right)\right] .
$$

At $t<0$, ac external bias $V_{\alpha}(t)$ or time-dependent part in Hamiltonian $\widetilde{V}_{\alpha}(t)$ is a constant and the system is in a steady state. Consequently, the total current is known from dc transport theory that is expressed in the form of Eq. (12) but with the Green's function and self-energy obtained from the orthogonal Hamiltonian defined above. Hence in the following we shall derive only the ac current when $t>0$. First, we shall look at the self-energy. From Eqs. (5) and (16),

$$
\begin{aligned}
\boldsymbol{\Sigma}_{\alpha}^{\gamma}\left(t, t^{\prime}\right)= & \mathfrak{W}_{\alpha}^{\dagger}(t)\left[\mathbf{T}_{c \alpha}^{0} \mathbf{g}_{\alpha \alpha}^{\gamma}\left(t, t^{\prime}\right) T_{\alpha c}^{0}\right] \mathfrak{W}_{\alpha}\left(t^{\prime}\right)=\mathfrak{W}_{\alpha}^{\dagger}(t) \\
& \times\left[\int \frac{d \epsilon}{2 \pi} e^{i \epsilon\left(t-t^{\prime}\right)} \Sigma_{\alpha}^{\gamma, e x}(\epsilon)\right] \mathfrak{W}_{\alpha}\left(t^{\prime}\right)=\mathfrak{W}_{\alpha}^{\dagger}(t) \mathfrak{V}_{\alpha}^{\dagger}(t) \\
& \times\left[\int \frac{d \epsilon}{2 \pi} e^{i \epsilon\left(t-t^{\prime}\right)} \Sigma_{\alpha}^{\gamma, 0}(\epsilon)\right] \mathfrak{V}_{\alpha}\left(t^{\prime}\right) \mathfrak{W}_{\alpha}\left(t^{\prime}\right),
\end{aligned}
$$

where $\mathfrak{V}_{\alpha}(t)=1$ for the downward pulse and $\mathfrak{V}_{\alpha}(t)=e^{i e V_{\alpha} t}$ for the upward pulse. Here $\boldsymbol{\Sigma}_{\alpha}^{\gamma, 0}(\boldsymbol{\epsilon})$ is the self-energy at zero bias, $\Sigma_{\alpha}^{\gamma, e x}(\epsilon)=\mathbf{T}_{c \alpha}^{0} \mathbf{g}_{\alpha \alpha}^{\gamma, e x}(\epsilon) \mathbf{T}_{\alpha c}^{0}$ is the self-energy at the unperturbed state defined above. In the downward case $\boldsymbol{\Sigma}_{\alpha}^{\gamma, e x}=\boldsymbol{\Sigma}_{\alpha}^{\gamma, 0}$; In the upward case $\boldsymbol{\Sigma}_{\alpha}^{\gamma, e x}=\boldsymbol{\Sigma}_{\alpha}^{\gamma, V}$. Setting $\mathbf{S}_{\alpha c}^{0}=\mathbf{S}_{c \alpha}^{0}=0, \boldsymbol{\Sigma}_{\alpha}^{\gamma, 0}$ and $\boldsymbol{\Sigma}_{\alpha}^{\gamma, V}$ 
are defined in Eq. (13) with zero and nonzero $V_{\alpha}$, respectively. We have $\boldsymbol{\Sigma}_{\alpha}^{r / a, V}(\epsilon)=\boldsymbol{\Sigma}_{\alpha}^{r / a, 0}\left(\epsilon-e V_{\alpha}\right)$. From Eqs. (17) and (18), we find

$$
\begin{aligned}
J_{\alpha}(t)= & 2 e \operatorname{Re} \int \frac{\mathrm{d} \epsilon}{2 \pi} \int_{-\infty}^{\mathrm{t}} \mathrm{dt}_{1} \mathrm{e}^{\mathrm{i} \epsilon\left(\mathrm{t}-\mathrm{t}_{1}\right)}\left[\mathbf{G}^{r}\left(t, t_{1}\right) \widetilde{\boldsymbol{\Sigma}}_{\alpha}^{<}\left(\epsilon, t_{1}, t\right)\right. \\
& \left.+\mathbf{G}^{<}\left(t, t_{1}\right) \widetilde{\boldsymbol{\Sigma}}_{\alpha}^{a}\left(\epsilon, t_{1}, t\right)\right],
\end{aligned}
$$

where the first term is the current flowing into the molecular device while the second one is the current flowing from the molecular device, and

$$
\tilde{\boldsymbol{\Sigma}}_{\alpha}^{\gamma}\left(\epsilon, t_{1}, t\right)=\mathcal{W}_{\alpha}^{\dagger}\left(t_{1}\right) \boldsymbol{\Sigma}_{\alpha}^{\gamma, 0}(\epsilon) \mathcal{W}_{\alpha}(t)
$$

where $\mathcal{W}_{\alpha}(t)=\mathfrak{V}_{\alpha}(t) \mathfrak{W}_{\alpha}(t)$. Here $\boldsymbol{\Sigma}_{\alpha \alpha}^{\gamma, 0}$ is the self-energy of lead- $\alpha$ at zero bias. The lesser Green's function is given by

$$
\begin{aligned}
\mathbf{G}^{<}\left(t, t^{\prime}\right)= & \int d t_{1} \int d t_{2} \mathbf{G}^{r}\left(t, t_{1}\right)\left[\sum_{\beta} \mathbf{\Sigma}_{\beta}^{<}\left(t_{1}, t_{2}\right)\right] \mathbf{G}^{a}\left(t_{2}, t^{\prime}\right)=\sum_{\beta} \int \frac{d \epsilon}{2 \pi} e^{-i \epsilon\left(t-t^{\prime}\right)}\left[\int_{-\infty}^{t} d t_{1} e^{i \epsilon\left(t-t_{1}\right)} \mathcal{W}_{\beta}(t) \mathbf{G}^{r}\left(t, t_{1}\right) \mathcal{W}_{\beta}^{\dagger}\left(t_{1}\right)\right] \mathbf{\Sigma}_{\beta}^{<, 0}(\boldsymbol{\epsilon}) \\
& \times\left[\int_{-\infty}^{t^{\prime}} d t_{2} e^{-i \epsilon\left(t^{\prime}-t_{2}\right)} \mathcal{W}_{\beta}\left(t_{2}\right) \mathbf{G}^{a}\left(t^{\prime}, t_{2}\right) \mathcal{W}_{\beta}^{\dagger}(t)\right]
\end{aligned}
$$

Substitute Eqs. (20) and (21) into Eq. (19) and introducing a spectrum function

$$
\mathbf{A}_{\alpha}(t, \epsilon)=\int_{-\infty}^{t} d t_{1} e^{i \epsilon\left(t-t_{1}\right)} \mathcal{W}_{\alpha}(t) \mathbf{G}^{r}\left(t, t_{1}\right) \mathcal{W}_{\alpha}^{\dagger}\left(t_{1}\right)
$$

we have

$$
\begin{gathered}
J_{\alpha}^{\text {in }}(t)=2 e \operatorname{Re} \int \frac{\mathrm{d} \epsilon}{2 \pi} \mathbf{A}_{\alpha}(\mathrm{t}, \epsilon) \mathbf{\Sigma}_{\alpha}^{<, 0}(\epsilon), \\
J_{\alpha}^{\text {out }}(t)=2 e \operatorname{Re} \int \frac{\mathrm{d} \epsilon}{2 \pi} \sum_{\beta} \mathbf{A}_{\beta}(\mathrm{t}, \epsilon) \mathbf{\Sigma}_{\beta}^{<, 0}(\epsilon) \widetilde{\mathbf{F}}_{\beta \alpha}(\mathrm{t}, \epsilon),
\end{gathered}
$$

where

$$
\begin{aligned}
\tilde{\mathbf{F}}_{\beta \alpha}(t, \epsilon)= & \int_{-\infty}^{t} d t^{\prime} e^{-i \epsilon\left(t-t^{\prime}\right)} \int \frac{d E}{2 \pi} \\
& \times e^{i E\left(t-t^{\prime}\right)} \mathbf{A}_{\beta}^{\dagger}\left(t^{\prime}, \boldsymbol{\epsilon}\right) \mathcal{W}_{\alpha}^{\dagger}\left(t^{\prime}\right) \boldsymbol{\Sigma}_{\alpha}^{a, 0}(E) \mathcal{W}_{\alpha}(t) .
\end{aligned}
$$

Very often, $\mathbf{\Sigma}^{r / a}\left(t-t^{\prime}\right)$ is singular at $t=t^{\prime}$, such as the quantum dot system with the wide-band limit $\boldsymbol{\Sigma}^{r / a}(0)$ $=\int \frac{d E}{2 \pi} \boldsymbol{\Sigma}^{r / a}(E)=\delta(0)(\mp \Gamma / 2)$, or the superconducting-quantum dot-normal metal system, and so on. In these cases, we should be careful with Eq. (25),

$$
\begin{aligned}
\widetilde{\mathbf{F}}_{\beta \alpha}(t, \epsilon)= & \mathbf{F}_{\beta \alpha}(t, \epsilon)+\overline{\mathbf{F}}_{\beta \alpha}(t, \epsilon) \\
= & \left(\int_{-\infty}^{t^{-}}+\frac{1}{2} \int_{t^{-}}^{t^{+}}\right) d t^{\prime} e^{-i \epsilon\left(t-t^{\prime}\right)} \int \frac{d E}{2 \pi} \\
& \times e^{i E\left(t-t^{\prime}\right)} \mathbf{A}_{\beta}^{\dagger}\left(t^{\prime}, \epsilon\right) \mathcal{W}_{\alpha}^{\dagger}\left(t^{\prime}\right) \mathbf{\Sigma}_{\alpha}^{a, 0}(E) \mathcal{W}_{\alpha}(t) .
\end{aligned}
$$

The first integral $\int_{-\infty}^{t^{-}}$is the same as Eq. (25), the second integral $\frac{1}{2} \int_{t^{-}}^{t^{+}}$now becomes $\overline{\mathbf{F}}_{\beta \alpha}(t, \epsilon)=\mathbf{A}_{\beta}^{\dagger}(t, \epsilon) \Delta_{\alpha}^{a}$, where we have defined

$$
\boldsymbol{\Delta}_{\alpha}^{r / a}=\frac{1}{2} \int_{t^{-}}^{t^{+}} d t^{\prime}\left[\int \frac{d E}{2 \pi} \boldsymbol{\Sigma}_{\alpha}^{r / a, 0}(E)\right]=\frac{1}{2} \int_{t^{-}}^{t^{+}} d t^{\prime} \mathbf{\Sigma}_{\alpha}^{r / a, 0}(0) .
$$

Then, Eq. (24) becomes

$$
\begin{aligned}
J_{\alpha}^{\text {out }}(t)= & 2 e \operatorname{Re} \int \frac{\mathrm{d} \epsilon}{2 \pi} \sum_{\beta} \mathbf{A}_{\beta}(\mathrm{t}, \epsilon) \mathbf{\Sigma}_{\beta}^{<, 0}(\epsilon) \mathbf{F}_{\beta \alpha}(\mathrm{t}, \boldsymbol{\epsilon}) \\
& +2 e \operatorname{Re} \int \frac{\mathrm{d} \epsilon}{2 \pi} \sum_{\beta} \mathbf{A}_{\beta}(\mathrm{t}, \epsilon) \mathbf{\Sigma}_{\beta}^{<, 0}(\epsilon) \mathbf{A}_{\beta}^{\dagger}(\mathrm{t}, \epsilon) \Delta_{\alpha}^{\mathrm{a}} .
\end{aligned}
$$

We note that Eq. (28) is the same as that derived in Ref. 5. Different from Ref. 5, we have split the expression into two terms. The first term corresponds to the nonwideband limit, i.e., when the linewidth function $\boldsymbol{\Gamma}$ goes to zero at large energy. The second term of Eq. (28) is related to the wideband limit. Hence, for a quantum dot with a Lorentzian linewidth function, ${ }^{5}$ only the first term is nonzero while for the system in contact with a superconducting lead both terms are nonzero.

So far, we have discussed the ac conduction current $J_{\alpha}(t)$ under the time-dependent bias derived from the evolution of the number operator of the electron in the semi-infinite lead- $\alpha$. Now we wish to address the issue of charge accumulation in the scattering region. In principle, this can be done by including the self-consistent Coulomb potential due to ac bias. ${ }^{27}$ However, at finite voltages, there is no close form expression for ac current if Coulomb potential is included. Alternatively, one can treat Coulomb potential phenomeno- 
logically as follows. From the continuity equation, $\Sigma_{\alpha} J_{\alpha}(t)$ $+d Q(t) / d t=0$, we see that the conduction current is not a conserved quantity. In the presence of ac bias, the displacement current $J_{\alpha}^{d}$ due to the charge pileup $d Q / d t$ inside the scattering region becomes important and must be considered. Since we have neglected the Coulomb interaction in our calculation, we can use the method of current partition ${ }^{31,32}$ to include the displacement current. This can be done by partitioning the total displacement current $\Sigma_{\alpha} J_{\alpha}^{d}=d Q / d t$ into each leads giving rise to a conserving total current $I_{\alpha}=J_{\alpha}+J_{\alpha}^{d}$. For symmetric systems like what we shall study below, it is reasonable to assume that $J_{L}^{d}=J_{R}^{d}$ from which we find $J_{\alpha}^{d}=-\left(J_{L}\right.$ $\left.+J_{R}\right) / 2$. Hence the total current is given by $I_{L}=\left(J_{L}-J_{R}\right) / 2$ (Ref. 24) which satisfies the current conservation $I_{L}+I_{R}=0$.

\section{TRANSIENT ac CURRENT}

Up to now, we have derived the general expression for time-dependent current, Eqs. (22), (23), (25), and (28) which can be used for orthogonal as well as nonorthogonal basis set. To calculate the transient current we have to solve the retarded Green's function $\mathbf{G}^{r}\left(t, t^{\prime}\right)$ and integrate it over time to find $\mathbf{A}_{\beta}(t, \epsilon)$ and $\widetilde{\mathbf{F}}_{\beta \alpha}(t, \boldsymbol{\epsilon})$. For the pulselike voltage $\tilde{V}_{\alpha}(t)= \pm \theta(-t)$, we can obtain the Green' function $\mathbf{G}^{r}\left(t, t^{\prime}\right)$ by solving Dyson equation $\mathbf{G}^{r}=\mathbf{G}^{r, e q}+\mathbf{G}^{r, e q} \Xi \mathbf{G}^{r}$ from the known history in the time domain. Depending on what is the chosen unperturbed system that can be solved exactly, the Dyson equation can be written in a different but equivalent form. In the study of time-dependent transport, it is better to treat the time independent, open steady state system as the unperturbed system as described in Sec. II A, and treat the time-dependent part $\widetilde{V}_{\alpha}(t)$ and $\mathbf{U}(t)$ as a perturbation. As a result, the effective self-energy $\Xi$, which is due to the ac bias, would have two sources: the perturbation in leads $\overline{\mathbf{\Sigma}}_{\alpha}^{r}$ and the induced Coulomb interaction in molecular device $\mathbf{U}(t)$. Then,

$$
\begin{aligned}
\mathbf{G}^{r}\left(t, t^{\prime}\right)= & \mathbf{G}^{r, e x}\left(t, t^{\prime}\right)+\int_{-\infty}^{0} d t_{1} \mathbf{G}^{r, e x}\left(t, t_{1}\right) \mathbf{U}\left(t_{1}\right) \mathbf{G}^{r}\left(t_{1}, t^{\prime}\right) \\
& +\int d t_{1} d t_{2} \mathbf{G}^{r, e x}\left(t, t_{1}\right)\left[\sum_{\alpha} \overline{\mathbf{\Sigma}}_{\alpha}^{r}\left(t_{1}, t_{2}\right)\right] \mathbf{G}^{r}\left(t_{2}, t^{\prime}\right),
\end{aligned}
$$

where $\mathbf{U}(t)$ is the response of the molecular device that is due to the Coulomb interaction when the time-dependent voltage is turned on. Here we have assumed an adiabatic response since most of time the variance of the applied electric field is much slower than the particles' intrinsic lifetime inside the scattering region. Then we have $\mathbf{U}(t)= \pm \mathbf{U} \theta(-t)$ for downward case and upward case with $\mathbf{U}=\mathbf{H}_{c}^{V}-\mathbf{H}_{c}^{0}$.

$$
\begin{gathered}
\int d t_{1} d t_{2}=\left(\int_{-\infty}^{0} d t_{1} \int_{-\infty}^{t_{1}} d t_{2}+\int_{0}^{t} d t_{1} \int_{-\infty}^{0} d t_{2}\right), \\
\overline{\mathbf{\Sigma}}_{\alpha}^{r}\left(t, t^{\prime}\right)=\boldsymbol{\Sigma}_{\alpha}^{r}\left(t, t^{\prime}\right)-\boldsymbol{\Sigma}_{\alpha}^{r, e x}\left(t-t^{\prime}\right) \\
\mathbf{\Sigma}_{\alpha}^{r, e x}\left(t-t^{\prime}\right)=\mathfrak{V}_{\alpha}^{\dagger}(t) \mathbf{\Sigma}_{\alpha}^{r, 0}\left(t-t^{\prime}\right) \mathfrak{V}_{\alpha}\left(t^{\prime}\right) .
\end{gathered}
$$

\section{A. Exact expression of $A_{\beta}(t, \epsilon)$ and $F_{\beta \alpha}(t, \epsilon)$}

Following the derivations in Ref. 5, we can get the exact expression for $\mathbf{A}_{\beta}(t, \boldsymbol{\epsilon})$ and $\mathbf{F}_{\beta \alpha}(t, \boldsymbol{\epsilon})$ with the aid of the expressions $\epsilon_{\beta}=\epsilon+e V_{\beta}$ and $\epsilon_{\beta \alpha}=\epsilon+e V_{\beta}-e V_{\alpha}$

$$
\begin{aligned}
\mathbf{A}_{\beta}^{D}(t, \epsilon)= & \mathbf{G}^{r, 0}(\boldsymbol{\epsilon})+\int \frac{d E}{2 \pi} e^{i(\epsilon-E) t} \mathbf{G}^{r, 0}(E)\left[Z\left(\epsilon_{\beta}\right)-Z(\epsilon)\right. \\
& \left.+\mathbf{P}_{D} \mathbf{G}^{r, V}\left(\epsilon_{\beta}\right)\right], \\
\mathbf{F}_{\beta \alpha}^{D}(t, \boldsymbol{\epsilon})= & \int \frac{d E}{2 \pi} Z^{*}(\epsilon) \mathbf{G}^{a, 0}(\epsilon) \mathbf{\Sigma}_{\alpha}^{a, 0}(E)+\int \frac{d E}{2 \pi} e^{-i(\epsilon-E) t} \\
& \times\left\{\left[Z^{*}\left(\boldsymbol{\epsilon}_{\beta}\right)-Z^{*}(\boldsymbol{\epsilon})+\mathbf{G}^{a, V}\left(\boldsymbol{\epsilon}_{\beta}\right) \mathbf{P}_{D}^{\dagger}\right] \mathbf{G}^{a, 0}(E) \mathbf{Q}_{D}(E)\right. \\
& \left.+\left[Z^{*}\left(\boldsymbol{\epsilon}_{\beta \alpha}\right) \mathbf{G}^{a, V}\left(\boldsymbol{\epsilon}_{\beta}\right)-Z^{*}(\epsilon) \mathbf{G}^{a, 0}(\boldsymbol{\epsilon})\right] \mathbf{\Sigma}_{\alpha}^{a, 0}(E)\right\},
\end{aligned}
$$

$$
\begin{aligned}
\mathbf{A}_{\beta}^{U}(t, \epsilon)= & \mathbf{G}^{r, V}\left(\epsilon_{\beta}\right)+\int \frac{d E}{2 \pi} e^{i\left(\epsilon_{\beta}-E\right) t} \times \mathbf{G}^{r, V}(E)\left[Z(\epsilon)-Z\left(\epsilon_{\beta}\right)\right. \\
& \left.+\mathbf{P}_{U} \mathbf{G}^{r, 0}(\epsilon)\right], \\
\mathbf{F}_{\beta \alpha}^{U}(t, \boldsymbol{\epsilon})= & \int \frac{d E}{2 \pi} Z^{*}\left(\epsilon_{\beta \alpha}\right) \mathbf{G}^{a, V}\left(\epsilon_{\beta}\right) \Sigma_{\alpha}^{a, 0}(E)+\int \frac{d E}{2 \pi} e^{-i\left(\epsilon_{\beta}-E\right) t} \\
& \times\left\{\left[Z^{*}(\epsilon)-Z^{*}\left(\epsilon_{\beta}\right)+\mathbf{G}^{a, 0}(\epsilon) \mathbf{P}_{U}^{\dagger}\right] \mathbf{G}^{a, V}(E) \mathbf{Q}_{U}(E)\right. \\
& \left.+e^{i e V_{\alpha} t}\left[Z^{*}(\epsilon) \mathbf{G}^{a, 0}(\epsilon)-Z^{*}\left(\epsilon_{\beta \alpha}\right) \mathbf{G}^{a, V}\left(\boldsymbol{\epsilon}_{\beta}\right)\right] \mathbf{\Sigma}_{\alpha}^{a, 0}(E)\right\},
\end{aligned}
$$

where

$$
\begin{gathered}
\mathbf{P}_{D}=Z\left(\epsilon_{\beta}\right) \mathbf{U}+\sum_{\delta}\left[Z\left(\epsilon_{\beta}\right)-Z\left(\epsilon_{\beta \delta}\right)\right]\left[\boldsymbol{\Sigma}_{\delta}^{r, 0}\left(\epsilon_{\beta \delta}\right)-\boldsymbol{\Sigma}_{\delta}^{r, 0}(E)\right] \\
\mathbf{P}_{U}=-Z(\epsilon) \mathbf{U}+\sum_{\delta}\left[Z(\epsilon)-Z\left(\epsilon_{\delta}\right)\right]\left[\boldsymbol{\Sigma}_{\delta}^{r, 0}(\epsilon)-\Sigma_{\delta}^{r, 0}\left(E-V_{\delta}\right)\right] \\
\mathbf{Q}_{D}(E)=\int \frac{d \epsilon^{\prime}}{2 \pi}\left[1-e^{i\left(\epsilon^{\prime}-E\right) t}\right] Z\left(\epsilon^{\prime}\right) \mathbf{\Sigma}_{\alpha}^{a, 0}\left(\epsilon^{\prime}\right) \\
\mathbf{Q}_{U}(E)=\int \frac{d \epsilon^{\prime}}{2 \pi}\left[1-e^{i\left(\epsilon_{\alpha}^{\prime}-E\right) t}\right] Z\left(\epsilon_{\alpha}^{\prime}\right) \boldsymbol{\Sigma}_{\alpha}^{a, 0}\left(\epsilon^{\prime}\right)
\end{gathered}
$$

with

$$
Z(\epsilon)=\left[i\left(E-\epsilon-i 0^{+}\right)\right]^{-1} .
$$

In the absence of the ac bias, the quantity $A_{\alpha}$ is the Fourier transform of the retarded Green's function while the quantity $F_{\beta \alpha}$ is related to the Fourier transform of the advanced Green's function. They are all expressed in terms of the unperturbed Green's functions $\mathbf{G}^{r / a, 0 / V}$ and self-energy $\boldsymbol{\Sigma}^{0 / V}$ which have been widely studied in molecular device using the NEGF-DFT formalism. $\mathbf{G}^{r / a, 0 / V}$ and self-energy $\mathbf{\Sigma}^{0 / V}$ can be expressed as

$$
\begin{gathered}
\mathbf{G}^{r / a, 0 / V}(\boldsymbol{\epsilon})=\left[\boldsymbol{\epsilon} \mathbf{I}-\mathbf{H}_{c}^{0 / V}-\boldsymbol{\Sigma}^{r / a, 0 / V}(\boldsymbol{\epsilon})\right]^{-1}, \\
\boldsymbol{\Sigma}_{\alpha}^{\gamma, 0}(\boldsymbol{\epsilon})=\left[\mathbf{T}_{c \alpha}^{0}-\epsilon \mathbf{S}_{c \alpha}^{0}\right] \mathbf{g}_{\alpha \alpha}^{\gamma}(\boldsymbol{\epsilon})\left[\mathbf{T}_{\alpha c}^{0}-\epsilon \mathbf{S}_{\alpha c}^{0}\right],
\end{gathered}
$$




$$
\boldsymbol{\Sigma}_{\alpha}^{\gamma, V}(\boldsymbol{\epsilon})=\left[\mathbf{T}_{c \alpha}^{0}-\epsilon^{\alpha} \mathbf{S}_{c \alpha}^{0}\right] \mathbf{g}_{\alpha \alpha}^{\gamma}\left(\epsilon^{\alpha}\right)\left[\mathbf{T}_{\alpha c}^{0}-\epsilon^{\alpha} \mathbf{S}_{\alpha c}^{0}\right],
$$

where $\gamma=r, a,<, \epsilon^{\alpha}=\epsilon-e V_{\alpha}$. Obviously, $\boldsymbol{\Sigma}_{\alpha}^{\gamma, V}(\epsilon)=\Sigma_{\alpha}^{\gamma, 0}(\epsilon$ $\left.-e V_{\alpha}\right)$. In the wideband limit, Eqs. (29)-(32) will reduce to the formula first derived by Jauho et al. ${ }^{24}$ With $\mathbf{A}$ and $\mathbf{F}$ obtained we can, in principle, solve the ac current biased by downward or upward pulse exactly. In practice, however, its computational cost is expensive for a realistic molecular device. For example, to calculate $J_{\alpha}^{o u t}(t)$, we have to do triple integrals over energy and repeat this procedure to collect data for all time sequence. In the numerical calculation especially in $a b$ initio modeling, it is practically very difficult if not impossible to calculate the transient current for the complex structure in molecular devices. So approximation must be made so that Eqs. (29)-(32) can be simplified.

\section{B. Approximate scheme of $\mathrm{A}_{\beta}(t, \epsilon)$ and $\mathrm{F}_{\beta \alpha}(t, \epsilon)$}

The approximate solution of $\mathbf{A}_{\beta}(t, \boldsymbol{\epsilon})$ and $\mathbf{F}_{\beta \alpha}(t, \epsilon)$ in Eqs. (29)-(32) have to satisfy the following requirements. First, it has to greatly reduce the calculational cost. Second, it has to keep essential physics of transient dynamics. Third, it must have the correct initial current at $t=0$ and approach the correct asymptotic limit at $t \rightarrow \infty$. The first goal is realized by eliminating double energy integral using a reasonable ansatz, with which the dynamical properties of molecular device is maintained.

To find such an ansatz, we first assume that $\mathbf{\Sigma}^{a, 0}(E)$ changes smoothly and slightly with $E$ and is analytic in the upper half plane, so that the typical integral like $\int d \epsilon d E \frac{e^{i(\epsilon-E) t}}{\left.-i(E-\epsilon+i)^{+}\right)} \mathbf{\Sigma}^{a, 0}(E)$ is roughly zero due to the different phase in $e^{l(E-E) t}$. Then the last term of $\mathbf{F}^{U / D}$ and the second term of $\mathbf{Q}^{U / D}$ disappear. Considering the following identity,

$$
\begin{aligned}
\int \frac{d E}{2 \pi} \frac{\boldsymbol{\Sigma}_{\alpha}^{a}(E)}{-i\left(E-\epsilon+i 0^{+}\right)}= & {\left[\int_{-\infty}^{0^{-}}+\frac{1}{2} \int_{0^{-}}^{0^{+}}\right] d \tau \boldsymbol{\Sigma}_{\alpha}^{a}(\tau) \int \frac{d E}{2 \pi} } \\
& \times \frac{e^{i E \tau}}{-i\left(E-\epsilon+i 0^{+}\right)} \\
& =\left[\int_{-\infty}^{0^{+}}-\frac{1}{2} \int_{0^{-}}^{0^{+}}\right] d \tau e^{i \epsilon \tau} \mathbf{\Sigma}_{\alpha}^{a}(\tau) \\
& =\mathbf{\Sigma}_{\alpha}^{a}(\epsilon)-\mathbf{\Delta}_{\alpha}^{a}
\end{aligned}
$$

and defining $\boldsymbol{\Sigma}_{\alpha}^{a}(E, \Delta)=\boldsymbol{\Sigma}_{\alpha}^{a}(E)-\boldsymbol{\Delta}_{\alpha}^{a}$, the first term of $\mathbf{F}_{U / D}$ and $\mathbf{Q}_{U / D}$ in Eq. (33) can be simplified, $\mathbf{F}_{U / D}$ now becomes

$$
\begin{aligned}
\mathbf{F}_{\beta \alpha}^{D} \simeq & \mathbf{G}^{a, 0}(\epsilon) \mathbf{\Sigma}_{\alpha}^{a, 0}(\epsilon, \Delta)+\int \frac{d E}{2 \pi} e^{-i(\epsilon-E) t}\left[Z^{*}\left(\epsilon_{\beta}\right)-Z^{*}(\epsilon)\right. \\
+ & \left.\mathbf{G}^{a, V}\left(\epsilon_{\beta}\right) \mathbf{P}_{D}^{\dagger}\right] \mathbf{G}^{a, 0}(E) \mathbf{\Sigma}_{\alpha}^{a, 0}(E, \Delta) \\
\mathbf{F}_{\beta \alpha}^{U} \simeq & \mathbf{G}^{a, V}\left(\boldsymbol{\epsilon}_{\beta}\right) \mathbf{\Sigma}_{\alpha}^{a, 0}\left(\epsilon_{\beta \alpha}, \Delta\right)+\int \frac{d E}{2 \pi} e^{-i\left(\epsilon_{\beta}-E\right) t} \\
& \times\left[Z^{*}(\epsilon)-Z^{*}\left(\epsilon_{\beta}\right)+\mathbf{G}^{a, 0}(\epsilon) \mathbf{P}_{U}^{\dagger}\right] \mathbf{G}^{a, V} \\
& \times(E) \mathbf{\Sigma}_{\alpha}^{a, 0}\left(E-e V_{\alpha}, \Delta\right) .
\end{aligned}
$$

We note that, in the wide-band limit, Eqs. (35) and (36) is exact. With our approximation we have eliminated one of the energy integrals in $J^{\text {out }}$, and $\mathbf{A}$ and $\mathbf{F}$ now have similar structures since $\widetilde{\mathbf{F}} \sim \mathbf{A}^{\dagger} \mathbf{\Sigma}^{a}$.

With the approximation defined in Eqs. (35) and (36), the current can be written in a compact form (see Sec. III C) if we introduce the effective Green's function

$$
\begin{aligned}
\widetilde{\mathbf{G}}^{r / a, 0}(E, \boldsymbol{\epsilon}) & =\left[E \mathbf{S}-\mathbf{H}_{c}^{0}-\sum_{\alpha} \boldsymbol{\Sigma}_{\alpha}^{r / a, 0}(\boldsymbol{\epsilon})\right]^{-1}, \\
\widetilde{\mathbf{G}}^{r / a, V}(E, \boldsymbol{\epsilon}) & =\left[E \mathbf{S}-\mathbf{H}_{c}^{V}-\sum_{\alpha} \boldsymbol{\Sigma}_{\alpha}^{r / a, V}(\boldsymbol{\epsilon})\right]^{-1} .
\end{aligned}
$$

In general we have to consider the overlap matrix $\mathbf{S}$. However, we should keep in mind that in the deriving of the time-dependent current, we have to orthogonalize the basis set, which would lead to $\mathbf{S}=\mathbf{I}$. Here $\widetilde{\mathbf{G}}^{r / a}(E, \boldsymbol{\epsilon})$ can be regarded as the Green's functions at energy $E$ and constant parameter $\boldsymbol{\epsilon}$ for open system with the effective Hamiltonian $\mathbf{H}_{e f f}^{r / a}=\mathbf{H}_{c}+\boldsymbol{\Sigma}_{\alpha}^{r}(\epsilon)$. For a given $\mathbf{H}_{e f f}$, Eqs. (37) and (38) are equivalent to

$$
\left(E \mathbf{S}-\mathbf{H}_{e f f}^{r}\right) \tilde{\mathbf{G}}^{r}=\mathbf{I} .
$$

On the other hand, Green's function can be expanded in terms of the eigenfunctions of the corresponding Hamiltonian,

$$
\widetilde{\mathbf{G}}^{r}=\sum_{n} \boldsymbol{\Psi}^{n} C_{n},
$$

where $\mathbf{H}_{e f f} \boldsymbol{\Psi}^{n}=E_{n}(\boldsymbol{\epsilon}) \boldsymbol{\Psi}_{n}$. Substituting Eq. (40) into Eq. (39), and using the general orthogonality relation $\boldsymbol{\Phi}^{n,+} \mathbf{S} \boldsymbol{\Psi}^{m}$ $=C_{m} \delta_{n m}$ [see Appendix $\left.\mathrm{A}\right]$ and the eigenvalue equation $\mathbf{H}_{\text {eff }} \boldsymbol{\Psi}^{n}=E_{n}(\epsilon) \boldsymbol{\Psi}^{n}$, we have

$$
\widetilde{\mathbf{G}}^{r}(E, \epsilon)=\sum_{n} \frac{\boldsymbol{\Psi}^{n} \boldsymbol{\Phi}^{n, \dagger}}{\left[E-E_{n}(\boldsymbol{\epsilon})\right] \boldsymbol{\Phi}^{n, \dagger} \mathbf{S} \boldsymbol{\Psi}^{n}} .
$$

Obviously, this Green's function can be calculated by finding the residues $\operatorname{Re~} \mathrm{s}_{n}=\boldsymbol{\Psi}^{n} \boldsymbol{\Phi}^{n, \dagger} / \boldsymbol{\Phi}^{n, \dagger} \mathbf{S} \boldsymbol{\Psi}^{n}$ at various poles $E$ $=E_{n}(\epsilon)$.

Then, we replace $Z(\epsilon) \mathbf{G}^{r / a}(E)$ in Eqs. (29), (31), (35), and (36) by $Z(\epsilon) \widetilde{\mathbf{G}}^{r / a}(E, \epsilon)$. Although $\widetilde{\mathbf{G}}^{r / a}(E, \boldsymbol{\epsilon})$ is different from initial Green's function $\mathbf{G}^{r / a}(E)=\left[E-\mathbf{H}_{c}-\boldsymbol{\Sigma}^{r / a}(E)\right]^{-1}$, this substitution is reasonable since the major contribution of the integration in Eqs. (29)-(32) comes from the pole $\epsilon$ in $Z(\epsilon)$ [see Eq. (34)]. Similarly, considering the major contribution of the pole of $Z(\epsilon)$, we replace $Z(\epsilon) \Sigma^{a, 0}(E)$ in Eqs. (29), (31), (35), and (36) by $Z(\epsilon) \mathbf{\Sigma}^{a, 0}(\epsilon)$. Since $\boldsymbol{\Sigma}(\epsilon)$ in $\widetilde{\mathbf{G}}^{r}(E, \epsilon)$ is independent of energy $E$, we can perform contour integration over energy $E$ in Eqs. (29) and (31) by closing a contour on lower half plane and perform the integration over energy $E$ in Eqs. (30) and (32) by closing a contour on upper half plane. Thus, energy integration over $E$ can be analytically performed. It should be noted that the self energy $\boldsymbol{\Sigma}^{r / a}$ is not independent of energy in contrast to the wide-band limit, this energy dependence is on $\epsilon$ but not on $E$. In this way, we can reduce the computational cost and keep the essential physics of the dynamics as we will show later. 


\section{Approximate expression of $A_{\beta}(t, \epsilon)$ and $F_{\beta \alpha}(t, \epsilon)$}

Now, considering the initial current and the asymptotic long time limit, we can write the approximate expression of $\mathbf{A}_{\beta}(t, \boldsymbol{\epsilon})$ and $\mathbf{F}_{\beta \alpha}(t, \boldsymbol{\epsilon})$ from Eqs. (29), (31), (35), and (36)

$$
\begin{gathered}
\mathbf{A}_{\beta}^{D / U}(t, \boldsymbol{\epsilon})=\mathbf{A}_{\beta, 1}^{D / U}+\mathbf{A}_{\beta, 2}^{D / U}, \\
\mathbf{F}_{\beta \alpha}^{D}(t, \boldsymbol{\epsilon})=\mathbf{A}_{\beta, 1}^{D, \dagger} \boldsymbol{\Sigma}_{\alpha}^{a, 0}\left(\epsilon_{\beta \alpha}, \Delta\right)+\mathbf{A}_{\beta, 2}^{D, \dagger} \boldsymbol{\Sigma}_{\alpha}^{a, 0}(\epsilon, \Delta), \\
\mathbf{F}_{\beta \alpha}^{U}(t, \epsilon)=\mathbf{A}_{\beta, 1}^{U, \dagger} \boldsymbol{\Sigma}_{\alpha}^{a, 0}(\epsilon, \Delta)+\mathbf{A}_{\beta, 2}^{U, \dagger} \boldsymbol{\Sigma}_{\alpha}^{a, 0}\left(\epsilon_{\beta \alpha}, \Delta\right)
\end{gathered}
$$

with

$$
\begin{gathered}
\mathbf{A}_{\beta, 1}^{D}=\int \frac{d E}{2 \pi} e^{i(\epsilon-E) t}\left\{Z\left(\epsilon_{\beta}\right) \widetilde{\mathbf{G}}^{r, 0}\left(E, \boldsymbol{\epsilon}_{\beta}\right)\left[\mathbf{I}+\Xi^{D} \mathbf{G}^{r, V}\left(\boldsymbol{\epsilon}_{\beta}\right)\right]\right\}, \\
\mathbf{A}_{\beta, 2}^{D}=\mathbf{G}^{r, 0}(\boldsymbol{\epsilon})-\int \frac{d E}{2 \pi} e^{i(\epsilon-E) t}\left[Z(\epsilon) \widetilde{\mathbf{G}}^{r, 0}(E, \boldsymbol{\epsilon})\right], \\
\mathbf{A}_{\beta, 1}^{U}=\int \frac{d E}{2 \pi} e^{i\left(\epsilon_{\beta}-E\right) t}\left\{Z(\epsilon) \widetilde{\mathbf{G}}^{r, V}(E, \boldsymbol{\epsilon})\left[\mathbf{I}+\Xi^{U} \mathbf{G}^{r, 0}(\boldsymbol{\epsilon})\right]\right\}, \\
\mathbf{A}_{\beta, 2}^{U}=\mathbf{G}^{r, V}\left(\boldsymbol{\epsilon}_{\beta}\right)-\int \frac{d E}{2 \pi} e^{i\left(\epsilon_{\beta}-E\right) t}\left[Z\left(\boldsymbol{\epsilon}_{\beta}\right) \widetilde{\mathbf{G}}^{r, V}\left(E, \boldsymbol{\epsilon}_{\beta}\right)\right],
\end{gathered}
$$

where

$$
\begin{gathered}
\Xi^{D}=\mathbf{U}+\sum_{\delta}\left[\boldsymbol{\Sigma}_{\delta}^{r, 0}\left(\boldsymbol{\epsilon}_{\beta \delta}\right)-\boldsymbol{\Sigma}_{\delta}^{r, 0}\left(\boldsymbol{\epsilon}_{\beta}\right)\right] \\
=\mathbf{U}+\sum_{\delta}\left[\boldsymbol{\Sigma}_{\delta}^{r, V}\left(\boldsymbol{\epsilon}_{\beta}\right)-\boldsymbol{\Sigma}_{\delta}^{r, 0}\left(\boldsymbol{\epsilon}_{\beta}\right)\right], \\
\Xi^{U}=-\mathbf{U}+\sum_{\delta}\left[\boldsymbol{\Sigma}_{\delta}^{r, 0}(\boldsymbol{\epsilon})-\Sigma_{\delta}^{r, 0}\left(\boldsymbol{\epsilon}-e V_{\delta}\right)\right] \\
=-\mathbf{U}+\sum_{\delta}\left[\boldsymbol{\Sigma}_{\delta}^{r, 0}(\boldsymbol{\epsilon})-\boldsymbol{\Sigma}_{\delta}^{r, V}(\boldsymbol{\epsilon})\right] .
\end{gathered}
$$

This is the second level of approximation. As we will see later that it is better than the first level approximation described below. Now we can make further approximation (the first level). To do this, we note that the Green's function $\mathbf{G}^{r}$ can be obtained using the Dyson equation,

$$
\mathbf{G}^{r, t o t}=\mathbf{G}^{r, e x}+\mathbf{G}^{r, e x} \Xi \mathbf{G}^{r, t o t},
$$

where $\mathbf{G}^{r}$, tot is the Green's function of system denoted by $\mathbf{H}^{t o t}=\mathbf{H}^{e x}+\mathbf{H}^{\prime}, \mathbf{G}^{r, e x}$ is the unperturbed Green's function corresponding to $\mathbf{H}^{e x}$ that can be exactly solved, $\boldsymbol{\Xi}$ is the effective self-energy describing $\mathbf{H}^{\prime}$. If we set $\mathbf{H}^{e x}$ and $\mathbf{H}^{\text {tot }}$ as zero biased open system and $V_{\alpha}$ biased open system, respectively, we have

$$
\mathbf{G}^{r, t o t}=\mathbf{G}^{r, V}(\boldsymbol{\epsilon})=\mathbf{G}^{r, 0}(\boldsymbol{\epsilon})+\mathbf{G}^{r, 0}(\boldsymbol{\epsilon}) \Xi^{D} \mathbf{G}^{r, V}(\boldsymbol{\epsilon}) .
$$

Similarly, if we treat $\mathbf{H}^{e x}$ and $\mathbf{H}^{\text {tot }}$ as $V_{\alpha}$ biased open system and zero biased open system, respectively, we obtain another Dyson equation

$$
\mathbf{G}^{r, t o t}=\mathbf{G}^{r, 0}(\boldsymbol{\epsilon})=\mathbf{G}^{r, V}(\boldsymbol{\epsilon})+\mathbf{G}^{r, V}(\boldsymbol{\epsilon}) \Xi^{U} \mathbf{G}^{r, 0}(\boldsymbol{\epsilon}) .
$$

Similar to the derivation of the second level of approximation, we can also replace $\mathbf{G}^{e x}(\boldsymbol{\epsilon})$ by $\widetilde{\mathbf{G}}^{e x}(E, \boldsymbol{\epsilon})$ in Eqs. (51) and (52) which leads to

$$
\begin{aligned}
& \widetilde{\mathbf{G}}^{r, V}(E, \boldsymbol{\epsilon}) \simeq \widetilde{\mathbf{G}}^{r, 0}(E, \boldsymbol{\epsilon})\left[\mathbf{I}+\Xi^{D} \mathbf{G}^{r, V}(\boldsymbol{\epsilon})\right], \\
& \widetilde{\mathbf{G}}^{r, 0}(E, \boldsymbol{\epsilon}) \simeq \widetilde{\mathbf{G}}^{r, V}(E, \boldsymbol{\epsilon})\left[\mathbf{I}+\Xi^{U} \mathbf{G}^{r, 0}(\boldsymbol{\epsilon})\right] .
\end{aligned}
$$

Then, Eqs. (45) and (47) can be further approximated as

$$
\begin{aligned}
\mathbf{A}_{\beta, 1}^{D} & =\int \frac{d E}{2 \pi} e^{i(\epsilon-E) t}\left[Z\left(\epsilon_{\beta}\right) \widetilde{\mathbf{G}}^{r, V}\left(E, \boldsymbol{\epsilon}_{\beta}\right)\right], \\
\mathbf{A}_{\beta, 1}^{U} & =\int \frac{d E}{2 \pi} e^{i\left(\epsilon_{\beta}-E\right) t}\left[Z(\epsilon) \widetilde{\mathbf{G}}^{r, 0}(E, \epsilon)\right] .
\end{aligned}
$$

This is the first level of approximation. It is easy to confirm that when the self-energy is energy independent these two approximations lead to exactly the same expression of transient current in the wide-band limit. In the next section we will numerically compare these two approximations with the exact solution.

\section{Initial and asymptotic currents}

We now show that the currents calculated from Eqs. (23), (28), and (42)-(48) and from Eqs. (23), (28), (42)-(44), (46), (48), (54), and (55) satisfy the correct current limit at initial $t=0$ and asymptotic limit $t \rightarrow \infty$ times. Note that the initial current and asymptotic currents can be calculated from a standard dc transport nonequilibrium Green's-function analysis. It is expected that the asymptotic current for the downward pulse $J_{\alpha}^{D}(t \rightarrow \infty)$ and initial current for the upward pulse $J_{\alpha}^{U}(t=0)$ are zero since there is no bias in the system. Now we discuss the limiting cases for two versions of approximations developed in Sec. III C.

When $t=0, e^{i(\epsilon-E) t}=1$, we can perform integration over energy $E$ in Eqs. (45)-(48) by closing a contour at upper half plane, where only a single residual exists at an energy pole of Z. At $t=0, \widetilde{\mathbf{G}}^{r / a, 0 / V}(E, \boldsymbol{\epsilon})=\mathbf{G}^{r / a, 0 / V}(\boldsymbol{\epsilon})$, therefore Eqs. (45) and (47) and Eqs. (54) and (55) are equivalent. Now we focus on the current obtained from Eqs. (23), (28), (42)-(44), (46), (48), (54), and (55). After integrating over $\epsilon$, the two terms in Eqs. (46) and (48) cancels to each other, then from Eqs. (54) and (55), $\mathbf{A}_{\beta}^{D / U}(t=0)$ becomes

$$
\begin{gathered}
\mathbf{A}_{\beta}^{D}(t=0)=\widetilde{\mathbf{G}}^{r, V}\left(\boldsymbol{\epsilon}_{\beta}, \boldsymbol{\epsilon}_{\beta}\right)=\mathbf{G}^{r, V}\left(\boldsymbol{\epsilon}_{\beta}\right), \\
\mathbf{A}_{\beta}^{U}(t=0)=\widetilde{\mathbf{G}}^{r, 0}(\boldsymbol{\epsilon}, \boldsymbol{\epsilon})=\mathbf{G}^{r, 0}(\boldsymbol{\epsilon}) .
\end{gathered}
$$

For $\mathbf{F}_{\beta \alpha}$, we can perform integration over energy $E$ by closing a contour at lower half plane. Similarly, there also exists only a single residual on energy pole $E_{Z}$ of $Z^{*}$ in the lower half plane, and

$$
\mathbf{F}_{\beta \alpha}^{D}(t=0)=\widetilde{\mathbf{G}}^{a, V}\left(\boldsymbol{\epsilon}_{\beta}, \boldsymbol{\epsilon}_{\beta}\right) \mathbf{\Sigma}_{\alpha}^{a, 0}\left(\boldsymbol{\epsilon}_{\beta \alpha}, \Delta\right)=\mathbf{G}^{a, V}\left(\boldsymbol{\epsilon}_{\beta}\right) \boldsymbol{\Sigma}_{\alpha}^{a, V}\left(\boldsymbol{\epsilon}_{\beta}, \Delta\right),
$$




$$
\mathbf{F}_{\beta \alpha}^{U}(t=0)=\widetilde{\mathbf{G}}^{a, V}(\epsilon, \epsilon) \boldsymbol{\Sigma}_{\alpha}^{a, 0}(\epsilon, \Delta)=\mathbf{G}^{a, 0}(\epsilon) \boldsymbol{\Sigma}_{\alpha}^{a, 0}(\epsilon, \Delta)
$$

Substituting Eqs. (56)-(59) into Eqs. (23) and (28), and considering

$$
\begin{gathered}
\boldsymbol{\Sigma}_{\beta}^{\gamma, 0}(\epsilon)=\boldsymbol{\Sigma}_{\beta}^{\gamma, V}\left(\epsilon_{\beta}\right), \\
\mathbf{G}^{<, 0 / V}(\boldsymbol{\epsilon})=\mathbf{G}^{r, 0 / V}(\epsilon)\left[\sum_{\beta} \boldsymbol{\Sigma}_{\beta}^{<, 0 / V}(\epsilon)\right] \mathbf{G}^{a, 0 / V}(\boldsymbol{\epsilon}), \\
\boldsymbol{\Sigma}_{\beta}^{<, 0}(\boldsymbol{\epsilon})=f(\epsilon)\left[\boldsymbol{\Sigma}_{\beta}^{a, 0}(\boldsymbol{\epsilon})-\boldsymbol{\Sigma}_{\beta}^{r, 0}(\boldsymbol{\epsilon})\right], \\
\boldsymbol{\Sigma}_{\beta}^{<, V}(\boldsymbol{\epsilon})=f\left(\epsilon-e V_{\beta}\right)\left[\boldsymbol{\Sigma}_{\beta}^{a, V}(\epsilon)-\boldsymbol{\Sigma}_{\beta}^{r, V}(\epsilon)\right],
\end{gathered}
$$

where $f(\epsilon)$ is Fermi distribution function, we have initial current at $t=0$

$$
\begin{gathered}
J_{\alpha}^{D}=2 e \operatorname{Re} \int \frac{\mathrm{d} \epsilon}{2 \pi} \mathbf{G}^{\mathrm{r}, \mathrm{V}}(\epsilon) \mathbf{\Sigma}_{\alpha}^{<, \mathrm{V}}(\epsilon)+\mathbf{G}^{<, \mathrm{V}}(\epsilon) \mathbf{\Sigma}_{\alpha}^{\mathrm{a}, \mathrm{V}}(\epsilon), \\
J_{\alpha}^{U}=2 e \operatorname{Re} \int \frac{\mathrm{d} \epsilon}{2 \pi} \mathbf{G}^{\mathrm{r}, 0}(\epsilon) \mathbf{\Sigma}_{\alpha}^{<, 0}(\epsilon)+\mathbf{G}^{<, 0}(\epsilon) \boldsymbol{\Sigma}_{\alpha}^{\mathrm{a}, 0}(\epsilon) .
\end{gathered}
$$

Equations (61) and (62) are the same as the formal dc current expression in the case of nonzero bias and zero bias, respectively. $J_{\alpha}^{U}(t=0)$ in Eq. (62) is exactly zero since the Fermi distribution in $\mathbf{\Sigma}_{\alpha}^{<}$and $\mathbf{G}^{<}$are equal for $\alpha=L$ and $\alpha=R$.

When $t \rightarrow \infty$, by virtue of the Riemann-Lebesgue lemma, ${ }^{33}$ the Fourier integral over $\epsilon$ vanishes, i.e., $\int \frac{d \epsilon}{2 \pi} e^{-i \epsilon t} \mathbf{G}^{r} \mathbf{\Sigma}^{r} \ldots$ equal to zero at $t \rightarrow \infty$ since there always exist poles in lower half plane. With this in mind, we have

$$
\begin{gathered}
\mathbf{A}_{\beta}^{D}(t \rightarrow \infty, \epsilon)=\mathbf{G}^{r, 0}(\epsilon), \\
\mathbf{F}_{\beta \alpha}^{D}(t \rightarrow \infty, \epsilon)=\mathbf{G}^{a, 0}(\epsilon) \Sigma_{\alpha}^{a, 0}(\epsilon, \Delta), \\
\mathbf{A}_{\beta}^{U}(t \rightarrow \infty, \epsilon)=\mathbf{G}^{r, V}\left(\epsilon_{\beta}\right), \\
\mathbf{F}_{\beta \alpha}^{U}(t \rightarrow \infty, \epsilon)=\mathbf{G}^{a, V}\left(\epsilon_{\beta}\right) \mathbf{\Sigma}_{\alpha}^{a, 0}\left(\epsilon_{\beta \alpha}, \Delta\right)=\mathbf{G}^{a, V}\left(\epsilon_{\beta}\right) \mathbf{\Sigma}_{\alpha}^{a, V}\left(\epsilon_{\beta}, \Delta\right) .
\end{gathered}
$$

From Eqs. (63)-(66) and Eqs. (23) and (28), we have the asymptotic current

$$
\begin{aligned}
& J_{\alpha}^{D}=2 e \operatorname{Re} \int \frac{\mathrm{d} \epsilon}{2 \pi} \mathbf{G}^{\mathrm{r}, 0}(\epsilon) \mathbf{\Sigma}_{\alpha}^{<, 0}(\epsilon)+\mathbf{G}^{<, 0}(\epsilon) \mathbf{\Sigma}_{\alpha}^{\mathrm{a}, 0}(\epsilon), \\
& J_{\alpha}^{U}=2 e \operatorname{Re} \int \frac{\mathrm{d} \epsilon}{2 \pi} \mathbf{G}^{\mathrm{r}, \mathrm{V}}(\epsilon) \mathbf{\Sigma}_{\alpha}^{<, \mathrm{V}}(\epsilon)+\mathbf{G}^{<, \mathrm{V}}(\epsilon) \Sigma_{\alpha}^{\mathrm{a}, \mathrm{V}}(\epsilon) .
\end{aligned}
$$

It is easy to see, Eqs. (67) and (68) are the formal dc current expression in the case of zero bias and nonzero bias, respectively, and $J_{\alpha}^{D}(t \rightarrow \infty)$ in Eq. (67) is exactly zero.

\section{COMPARISON WITH THE EXACT RESULT IN QUANTUM DOT SYSTEM}

Now we consider a system composed of a single-level quantum dot connected to external leads with a Lorentzian linewidth. This system can be solved exactly to give a transient current for pulselike bias. ${ }^{5}$ We can obtain transient current using three methods: (i) the exact current expressed by Eqs. (23) and (28)-(32), (ii) the first level of approximation from Eqs. (23), (28), (42)-(44), (46), (48), (54), and (55) and (iii) the second level of approximation from Eqs. (23), (28), and (42)-(48). We will compare the current obtained from these three methods. The system is described by the following simple Hamiltonian

$$
H=\sum_{k_{\alpha}} \epsilon_{k_{\alpha}}(t) c_{k_{\alpha}}^{\dagger} c_{k_{\alpha}}+\epsilon_{d}(t) d^{\dagger} d+\sum_{k_{\alpha}}\left(t_{k_{\alpha}} c_{k_{\alpha}}^{\dagger} d+\text { h.c. }\right),
$$

where $\epsilon_{d}(t)=\epsilon_{d}^{0}+U(t)$ and $\epsilon_{k_{\alpha}}(t)=\epsilon_{k_{\alpha}}^{0}+V_{\alpha}(t)$. Because the scattering region has only one state with energy level $\epsilon_{d}^{0}$, the Green's functions $G(\epsilon)$ and self-energy $\Sigma(\epsilon)$ thus become scalars instead of matrices. If we choose linewidth function $\Gamma_{\alpha}(\omega) \equiv 2 \pi \rho_{\alpha}(\omega)\left|t_{k_{\alpha}}\right|^{2}$ to be Lorentzian with the linewidth amplitude $\Gamma_{\alpha}^{0}$,

$$
\Gamma_{\alpha}(\omega)=\frac{W^{2}}{\omega^{2}+W^{2}} \Gamma_{\alpha}^{0}
$$

then $G^{\gamma}(\epsilon)$ and $\Sigma^{\gamma}(\epsilon)$ can be expressed as

$$
\begin{gathered}
G^{r / a, 0}(\epsilon)=\left[\epsilon-\epsilon_{d}^{0}-\sum_{\alpha} \Sigma^{r / a, 0}(\epsilon)\right]^{-1}, \\
G^{r / a, V}(\epsilon)=\left[\epsilon-\epsilon_{d}^{0}-U^{V}-\sum_{\alpha} \Sigma^{r / a, V}(\epsilon)\right]^{-1} \\
G^{<, 0 / V}(\epsilon)=G^{<, 0 / V}(\epsilon)\left[\sum_{\alpha} \Sigma^{<, 0 / V}(\epsilon)\right] G^{<, 0 / V}(\epsilon), \\
\Sigma_{\alpha}^{r / a, 0}(\epsilon)=\int \frac{d \omega}{2 \pi} \Gamma_{\alpha}(\omega) /\left(\epsilon-\omega \pm i 0^{+}\right), \\
\Sigma_{\alpha}^{r / a, V}(\epsilon)=\int \frac{d \omega}{2 \pi} \Gamma_{\alpha}(\omega) /\left(\epsilon-e V_{\alpha}-\omega \pm i 0^{+}\right), \\
\Sigma_{\alpha}^{<, 0}(\epsilon)=f(\epsilon)\left[\Sigma_{\alpha}^{a, 0}(\epsilon)-\Sigma_{\alpha}^{r, 0}(\epsilon)\right], \\
\Sigma_{\alpha}^{<, V}(\epsilon)=f\left(\epsilon-e V_{\alpha}\right)\left[\Sigma_{\alpha}^{a, V}(\epsilon)-\Sigma_{\alpha}^{r, V}(\epsilon)\right] .
\end{gathered}
$$

Using the theorem of residual, we can analytically perform integral in $A_{\beta}$ and $F_{\beta \alpha}$ for either exact formula or two approximate formulas. In the calculation, we set $\Gamma=\Gamma_{L}^{0}+\Gamma_{R}^{0}$ as the energy unit, and set $\Gamma_{L}^{0}=\Gamma_{R}^{0}=0.5$.

We first consider the transient current induced by opposite voltage $V_{L}(t)=-V_{R}(t)$. In this case, the equilibrium coulomb potential in quantum dot $U^{0, V}=0$, and the time-dependent perturbation coming from coulomb response $U(t)$ is assumed to be zero. It is a reasonable assumption since the coulomb potential in scattering region is canceled by the opposite 

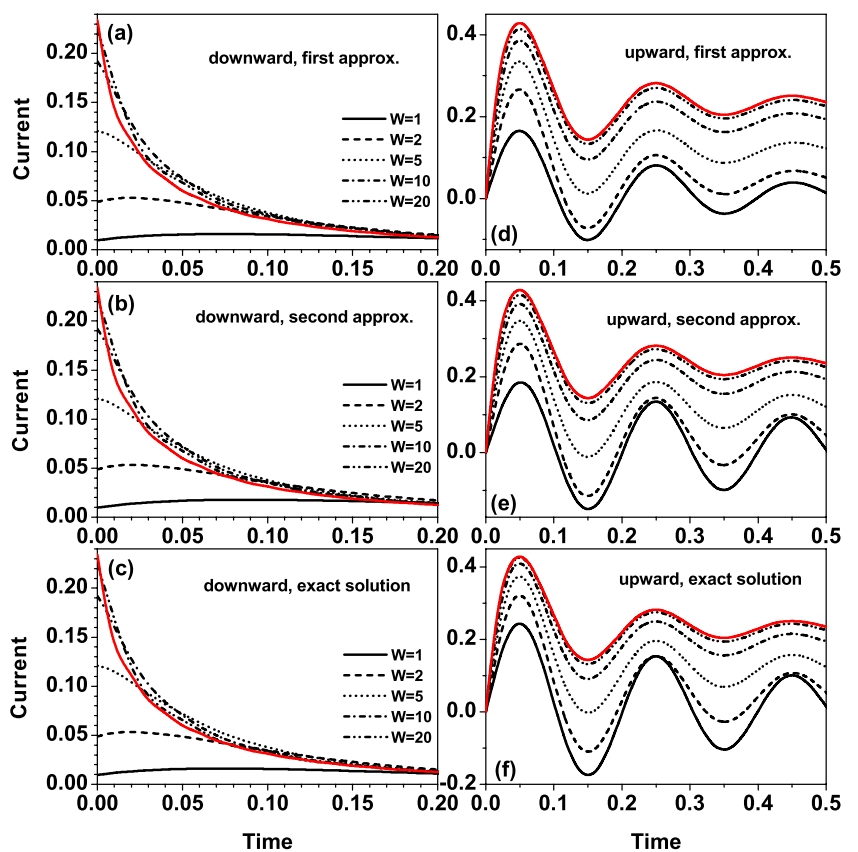

FIG. 1. (Color online) Time-dependent current $J(t)$ corresponding to an opposite downward pulse or upward pulse in three versions: the exact solution and two approximations. The different black lines are for different bandwidth $W$. The red (gray in print) line is for $W=\infty$, i.e., the wide-band limit. The current is in the unit of $e \Gamma$, and the time is in the unit of $2 \pi / \Gamma . e V_{L}=-e V_{R}=5$.

voltage in left and right lead. In Fig. 1, we plot two approximated transient currents and exact transient current in downward [panels (a), (b), and (c)] and upward [panels (d), (e), and (f)] case vs time for different bandwidth $W$. We find that for all bandwidth $W$, the approximated current and exact cur-

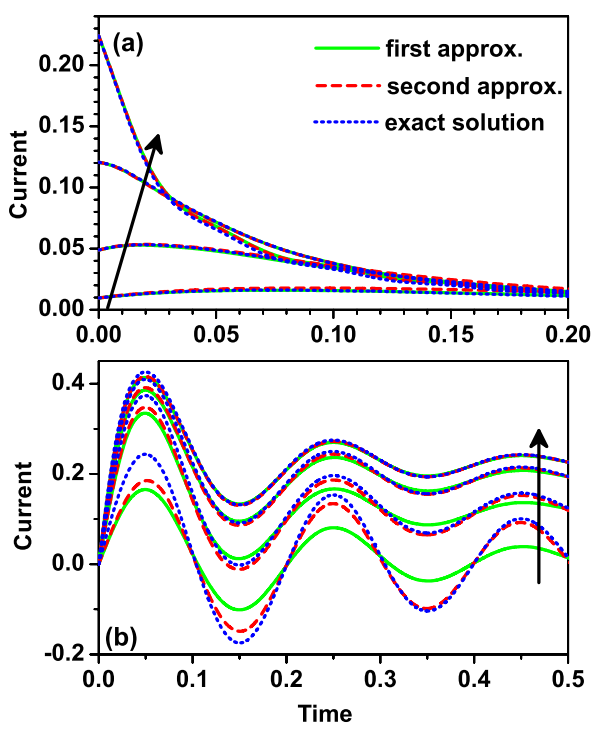

FIG. 2. (Color online) Merged version of Fig. 1 for $W=1,2,5$, and 20. Panel (a) corresponding to the downward pulse current comes from panels (a), (b), and (c) in Fig. 1, panel (b) corresponding to upward pulse current comes from panels (d), (e), and (f). Along the black arrow, the bandwidth are $W=1,2,5$, and 20, respectively.

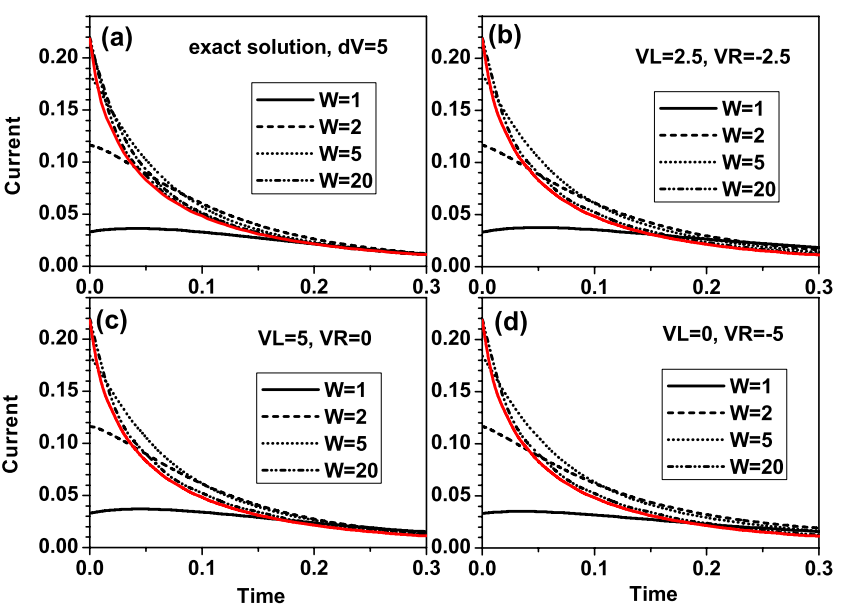

FIG. 3. (Color online) Panel (a): exact time-dependent current $J(t)$ corresponding to downward pulse for $d V=V_{L}-V_{R}=5$. Panels (b)-(d) are corresponding to the second approximate transient current corresponding to downward pulse for opposite voltage $V_{L}=$ $-V_{R}=2.5$, asymmetric voltage $V_{L}=5, V_{R}=0$ and $V_{L}=0, V_{R}=-5$, respectively. The different black lines are for different bandwidths $W$. The red (gray in print) line is wide-band limit for $W=\infty$.

rent have the same dynamical behaviors. Figure 2 gives direct comparison where we merge panels (a), (b), and (c) in Fig. 1 as panel (a) in Fig. 2, and merge panels (d), (e), and (f) in Fig. 1 as panel (b) in Fig. 2. We can see that for the downward pulse [panel (a)], transient current using three formulas are almost indistinguishable. This means that in the opposite voltage, our approximation, the first approximation [Eqs. (46), (48), (54), and (55)] and the second approximation [Eqs. (45)-(48)] are all very good for studying transient dynamics. For the upward pulse, although the approximations are not as good as in downward case, the currents calculated from approximate scheme are still in good agreement with the exact solution especially for the second approximation. Hence we may conclude that the two approximations are all reasonable in the opposite voltage $V_{L}(t)=-V_{R}(t)$. They can be used to study transient dynamics in the real molecular device to speed up the calculation.

Next, we focus on the asymmetric voltage, i.e., $V_{L}(t)$ $\neq V_{R}(t)$. In this case, the equilibrium coulomb potential in quantum dot $U^{0 / V}$, and the time-dependent perturbation coming from coulomb response $U(t)$ cannot be canceled by the voltage in left and right lead. In principle, perturbation $U(t)$ should be calculated by solving time-dependent Schrödinger equation, it will be very difficult and computational demanding therefore cannot be implemented in real molecular device. As an alternative scheme, we have set $U(t)$ $=\left[e V_{L}(t) \Gamma_{L}^{0}+e V_{L}(t) \Gamma_{L}^{0}\right] / \Gamma$. For the single level quantum dot system, this is exact because the central scattering region now is expressed in a scalar instead of matrices, which leads to the same transient current for the opposite voltage $V_{L}(t)$ $=-V_{R}(t)$ and asymmetric voltage $V_{L}(t)=V(t), V_{R}(t)=0$ or $V_{L}(t)=0, V_{R}(t)=-V(t)$ in the exact solution.

For the first approximation the poles in time-dependent term $e^{i(\epsilon-E) t}$ are different from that in the second level approximation, i.e., the poles of $\widetilde{\mathbf{G}}^{r, 0}$ in Eq. (45) and $\widetilde{\mathbf{G}}^{r, V}$ in Eq. (47) are replaced by the poles of $\widetilde{\mathbf{G}}^{r, V}$ in Eq. (54) and $\widetilde{\mathbf{G}}^{r, 0}$ in 


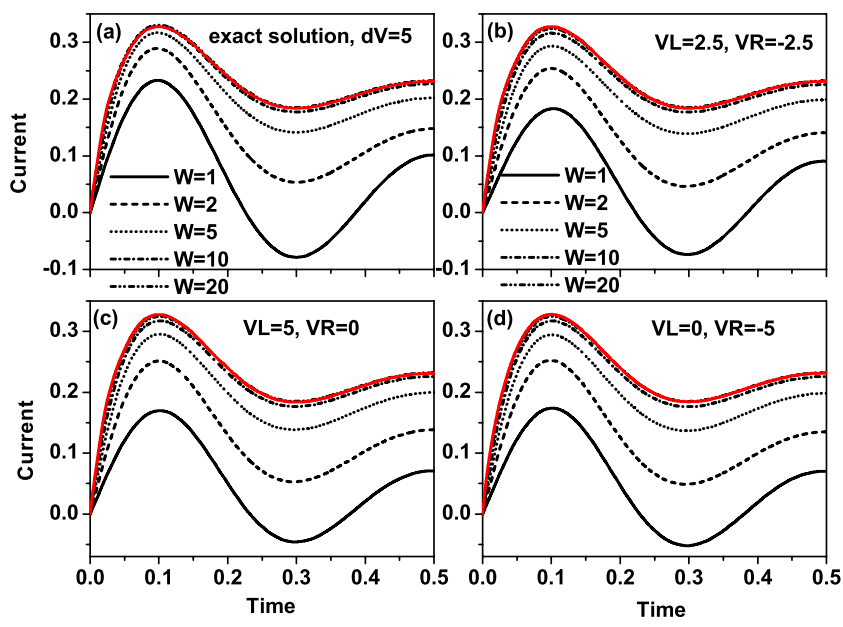

FIG. 4. (Color online) Same to Fig. 3, transient current corresponding to upward pulse vs time are plotted.

Eq. (55), respectively. Because of this, the time evolution process are not as accurate in the first approximation, especially for the large $V_{\alpha}$. So, for the asymmetric voltage, the second approximation is better. In Figs. 3 and 4, we compare the transient current obtained from the second approximation [panels (b)-(d)] for opposite or asymmetric voltage with the exact transient current [panel (a)] in response to the downward pulse and upward pulse, respectively. We find that all transient currents from the second approximation in Figs. 3 and 4 [panel (b)] are very close to the exact result [panel (a)]. Moreover, in Figs. 3 and 4, the approximate transient current in panels (b), (c), and (d) have almost the same behavior. It is safe to say that our approximations have kept essential physics of dynamical transport properties.

\section{SEVERAL EXAMPLES FOR REAL MOLECULAR DEVICES}

In this section, we implement our approximate formula in two representative molecular devices including a short carbon chain coupled to aluminum leads and a $C_{60}$ molecule coupled to aluminum leads. These systems were chosen because they are typical in first-principles calculation and their practical importance to nanoelectronics. In Figs. 5(a) and $5(\mathrm{~b})$, we show the structure of $\mathrm{Al}-\mathrm{C}_{5}-\mathrm{Al}$ and $\mathrm{Al}-\mathrm{C}_{60}-\mathrm{Al}$, respectively, where $\mathrm{Al}$ leads are along (100) direction, one unit

(a)
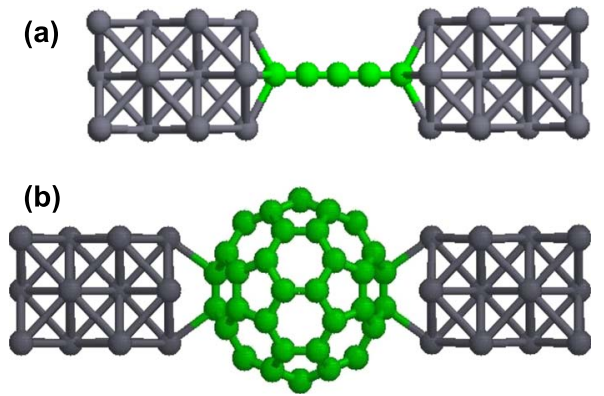

FIG. 5. (Color online) Panel (a): structure of Al-C5-Al. Panel (b): structure of $\mathrm{Al}-\mathrm{C}_{60}-\mathrm{Al}$.
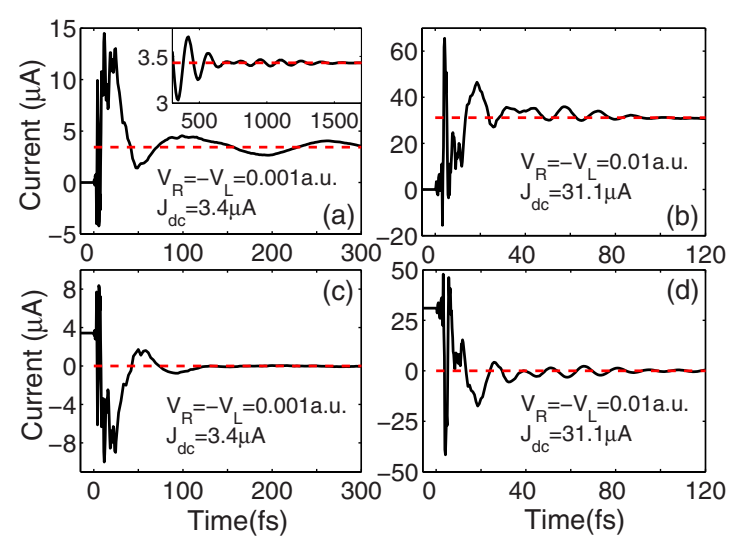

FIG. 6. (Color online) Time-dependent current $J(t)$ corresponding to the upward pulse [panels (a) and (b)] and the downward pulse [panels (c) and (d)] for different external voltages $V_{\alpha}$ in $\mathrm{Al}-\mathrm{C}_{5}-\mathrm{Al}$ device. The inset of panel (a) shows the long time behavior of the time-dependent current. The red (gray in print) dashed lines in panels indicate asymptotic current $J(t \rightarrow \infty)$ which the dc current biased by $V_{L / R}$ labeled in corresponding panels for the upward pulse, and arrive at zero for the downward case.

cell of $\mathrm{Al}$ lead consists of $9 \mathrm{Al}$ atoms and total 40 atoms were included in the simulation box. For the Al-C5-Al device, the nearest distance between $\mathrm{Al}$ leads and the carbon chain is 3.781 a.u. and the distance of $\mathrm{C}-\mathrm{C}$ bond is 2.5 a.u. (1 a.u. $=0.529 \AA$ ). In Al-C60-Al device, the distance between the $\mathrm{Al}$ atom and the nearest $\mathrm{C}$ atom equal to 3.625 a.u.

To calculate the dynamic response of molecular devices, we have used the first-principles quantum transport package MATDCAL. ${ }^{34}$ Considering the complicated coulomb response in scattering region, we set $V_{L}(t)=-V_{R}(t)$. In this case, the first approximation is simple but as good as the second one. So, in the following, the first approximate formula [Eqs. (23), (28), (42)-(44), (46), (48), (54), and (55)] is used. In principle, the calculation involves the following steps: (1) calculate the device Hamiltonian including central scattering Hamiltonian and lead Hamiltonian using NEGF-DFT package to get two potential landscapes $U^{0}$ at zero bias and $U^{V}$ at $V_{\alpha}$ bias, respectively. They are originally expressed in a nonorthogonal fireball basis. (2) Orthogonalize the nonorthogonal device Hamiltonian using the approach ${ }^{35}$ introduced in Appendix B so that they are finally expressed in an orthogonal basis. (3) With the orthogonal lead Hamiltonian $H_{\alpha}$, one calculates zero biased self energy $\boldsymbol{\Sigma}_{\alpha}^{r / a, 0}$ and $V_{\alpha}$ biased self energy $\boldsymbol{\Sigma}_{\alpha}^{r / a, V}$ from Eqs. (13) and (14) using the transfer matrix method. ${ }^{30}$ (4) With orthogonalized central scattering Hamiltonian $\mathbf{H}_{c}^{0}$ and $\mathbf{H}_{c}^{V}$ and self-energy $\boldsymbol{\Sigma}_{\alpha}^{r / a, 0}$ and $\boldsymbol{\Sigma}_{\alpha}^{r / a, V}$ obtained from two potential landscapes $U^{0}$ and $U^{V}$, one solves the effective Green's function $\mathbf{G}^{r / a, 0 / V}$ using Eqs. (37) and (38) by calculating its poles and residuals from Eq. (41). Step (1)-(4) are time independent processes and easy to perform. (5) Calculate time-dependent quantities $\mathbf{A}_{\beta, 1}^{D / U}$ and $\mathbf{A}_{\beta, 2}^{D / U}$ from Eqs. (54) and (55) and Eqs. (46) and (48). Then $\mathbf{A}_{\beta}$ and $\mathbf{F}_{\beta \alpha}$ can be calculated from Eqs. (42)-(44). (6) Integrate over $\epsilon$ and obtain the final ac current $J^{D / U}(t)=\left[J_{L}^{D / U}(t)\right.$ $\left.-J_{R}^{D / U}(t)\right] / 2$ from Eqs. (23) and (28).

First we study the $\mathrm{Al}-\mathrm{C}_{5}$-Al structure. In Fig. 6, we plot the transient current $J(t)$ corresponding to the upward pulse 


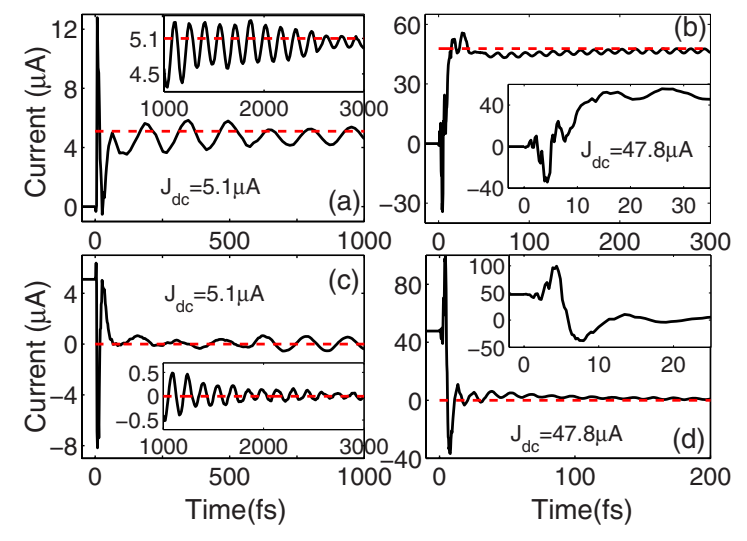

FIG. 7. (Color online) Time-dependent current $J(t)$ corresponding to the upward pulse [panels (a) and (b)] and the downward pulse [panels (c) and (d)] in Al- $\mathrm{C}_{60}-\mathrm{Al}$ device for different $V_{\alpha}$. In panels (a) and (c), $V_{R}=-V_{L}=0.001$ a.u.. In panels (b) and (d), $V_{R}=-V_{L}$ $=0.01$ a.u.. Same to Fig. 6, the red (gray in print) dashed lines in panels indicate asymptotic current $J(t \rightarrow \infty)$. The long time ac current or detailed short time ac current are shown in inset of panels.

[panels (a) and (b)] and the downward pulse [panels (c) and (d)] for different external voltages $V_{R}=-V_{L}=0.001$ a.u. [panels (a) and (c)] and $V_{R}=-V_{L}=0.01$ a.u. [panels (b) and (d)] in $\mathrm{Al}-\mathrm{C}_{5}-\mathrm{Al}$ structure. Following observations are in order: (1) as we have discussed in Sec. III D, for all bias voltages $V_{\alpha}$ the transient currents indeed reach the correct limits at $t=0$ and $t \rightarrow \infty$. For the upward pulse, $J(t=0)=0$ and $J(t$ $\rightarrow \infty)=J_{\mathrm{dc}}$ while for the downward pulse we have $J(t=0)$ $=J_{\mathrm{dc}}$ and $J(t \rightarrow \infty)=0$. (2) For both upward pulse (turn-on voltage) and downward pulse (turn-off voltage), once the bias voltage is switched, currents oscillate rapidly in the first a few or tens femtoseconds and then gradually approach to the steady-state values, i.e., $J_{d c}$ for turn-on voltage and zero for turn-off voltage. The larger the voltage $V_{\alpha}$, the more rapid the current oscillates. (3) Concerning the long time behavior, the time-dependent current oscillates with a frequency proportional to $\left|V_{\alpha}\right| .^{21}$ This is because the timedependent term $e^{i(\epsilon-E) t}$ in Eqs. (46), (48), (54), and (55) are $V_{\alpha}$ dependent. For the upward pulse, $e^{i\left(\epsilon_{\alpha}-E\right) t} \propto e^{i V_{\alpha} t}$, which directly leads to the oscillating frequency proportional to $\left|V_{\alpha}\right|$. For the downward pulse, although $e^{i(\epsilon-E) t}$ is $V_{\alpha}$ independent, in the energy integral on $E$, the pole $E_{n}$ of $\widetilde{\mathbf{G}}^{r}(E, \boldsymbol{\epsilon})$ are determined by the self-energy $\boldsymbol{\Sigma}_{\alpha}^{r, V}$. Since $\boldsymbol{\Sigma}_{\alpha}^{r, V}$ depends on $V_{\alpha}$, this leads to $V_{\alpha}$ dependent oscillating frequency. In addition, we notice that although the properties of dc conductance of short carbon chains are different for the chains with odd and even number atoms ${ }^{36}$ due to the completely different electronic structure near Fermi level, the ac signals are similar (see Ref. 21 where $\mathrm{Al}-\mathrm{C}_{4}-\mathrm{Al}$ structure was analyzed). This indicates that in ac transport, all states with energy from $-\infty$ to the Fermi energy are contributing, which is very different from dc case where only the states near Fermi level contribute to transport processes.

Next, we study the second sample: the $\mathrm{Al}-\mathrm{C}_{60}-\mathrm{Al}$ structure. In Fig. 7, the transient current $J(t)$ of the structure corresponding to an upward pulse [panels (a) and (b)] and a downward pulse [panels (c) and (d)] for different external voltages $V_{R}=-V_{L}=0.001$ a.u. [panels (a) and (c)] and $V_{R}=$
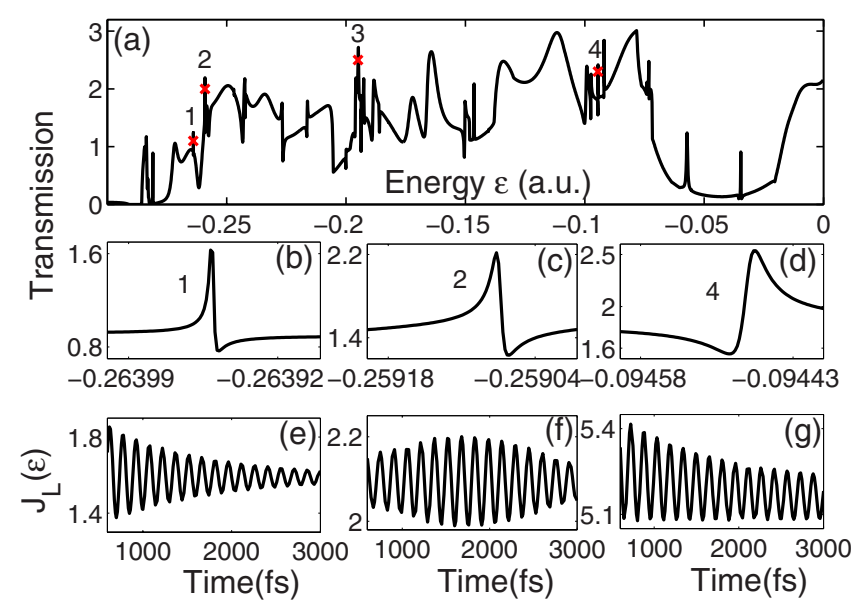

FIG. 8. (Color online) Panel (a): transmission coefficient $T(\epsilon)$ in the energy range from the energy band bottom to the Fermi energy. In the whole energy range, there are some resonant states corresponding to the very sharp transmission coefficient $T(\epsilon)$, as we have indicated (see red (gray in print) cross) and labeled (by 1, 2, 3, and 4) in panel (a), some of them contribute to the current at long time. We amplify the first, second, and fourth labeled resonant transmission in panels (b), (c), and (d), respectively. In panels (e)-(g), we plot the long time behavior of current $J_{L}(\epsilon)$ at a fixed $\epsilon$ for the first, second, and fourth resonant states. The external voltage $V_{\alpha}$ $=0.001$ a.u.

$-V_{L}=0.01$ a.u. [panels (b) and (d)] are plotted. Similar to the $\mathrm{Al}-\mathrm{C}_{5}-\mathrm{Al}$ structure, correct initial current $J(t=0)$ and asymptotic current $J(t \rightarrow \infty)$ are also obtained in $\mathrm{Al}-\mathrm{C}_{60}-\mathrm{Al}$ structure. In addition, there are also rapidly oscillations at short times after the switch although the oscillation is not as rapid as that in the $\mathrm{Al}-\mathrm{C}_{5}-\mathrm{Al}$ structure. Furthermore, similar to $\mathrm{Al}-\mathrm{C}_{5}-\mathrm{Al}$ structure, in gradually reaching the steady-state values, the current oscillates with a frequency proportional to $\left|V_{\alpha}\right|$ but its decay rate is much slower than that in $\mathrm{Al}-\mathrm{C}_{5}-\mathrm{Al}$ structure. It indicates that there are much more quasiresonant state that contribute to the transient current in $\mathrm{Al}-\mathrm{C}_{60}-\mathrm{Al}$ structure which is reasonable considering the complex electronic structure of isolated $\mathrm{C}_{60}$. In the following, we will analyze in detail how the current decays for the $\mathrm{Al}-\mathrm{C}_{60}-\mathrm{Al}$ structure.

Physically, decay time of current corresponds to the width of the quasibound state. In molecular devices, because the linewidth function $\Gamma(\epsilon)$ are complex and energy dependent matrix, we cannot extract characteristic time scale directly from $1 / \boldsymbol{\Gamma}$. As such, the transmission coefficient $T(\epsilon)$ is needed to understand the resonant state and corresponding characteristic time scale. In Fig. 8(a), we plot transmission coefficient $T(\epsilon)$ in the energy range from the energy band bottom to the Fermi energy for $\mathrm{Al}-\mathrm{C}_{60}-\mathrm{Al}$ structure at zero bias. Here, the sharp peaks [some of them, see red (gray in print) crossed signed peaks in Fig. 8(a)] correspond to resonant states with large lifetimes. At a particular resonant state, the incoming electron can dwell for a long time, which contributes to a much more slowly decaying current than other nonresonant states. In Figs. 8(b)-8(d), we amplify the first, second, and fourth labeled quasiresonant transmission, respectively, where the peaks' width $\Gamma_{\text {peak }} \sim 10^{-5}$ a.u. are in- 
dicated, corresponding to a decay time $\tau \sim 2400$ fs from the expression $\Gamma_{\text {peak }} t=1$. In Figs. $8(\mathrm{e})-8(\mathrm{~g})$, corresponding to different $\epsilon$ where the resonant peaks in Figs. 8(b)-8(d) are located, we plot long time behavior of current element $J_{L}(\epsilon)$. Here $J_{L}(\epsilon)$ is the time-dependent current for each energy $\epsilon$, the integration over which gives the final current $J_{\alpha}(t)$. We can see that for each resonant state the current $J_{L}(\epsilon)$ keeps oscillating in a long time comparable to the decay time $\tau$ $\sim 2400$ fs. Furthermore the intensity of the oscillation $\Delta J$ $\sim 0.2 \mu A$ is not very small comparing to the dc signal $J_{\mathrm{dc}}$ $=5.1 \mu A$.

After integration over energy, these slowly decaying currents $J_{L}(\epsilon)$ due to the resonant states may cancel to each other partially due to the difference in their phases. However, we should keep in mind that it is these resonant peaks that may give rise to convergence problem. Hence in the calculation, we should first scan the equilibrium and nonequilibrium transmission coefficient $(100,000$ energy points, for example) to resolve sharp resonant peaks in the whole energy range from minimum energy to Fermi energy. Then, for each sharp resonant peak, enough (100, for example) energy points should be chosen to converge the integration of the current $J_{L}(\epsilon)$ over $\epsilon$, i.e., $\int d \epsilon J(\epsilon)$. For the nonresonant state, i.e., the smoothly changed region in $T(\epsilon)$, the current $J(\epsilon)$ are integrated using less energy points.

As we have discussed that the resonant states are important for the transient current and they must be carefully treated in calculation. However, in the calculation of the effective Green's function $\widetilde{\mathbf{G}}^{r / a, 0 / V}$, a small imaginary part that is usually added to the real energy $\epsilon \rightarrow \epsilon+i \eta$ to help resolving the retarded or advanced self-energies. This in turn introduces pseudo resonant states. In order to eliminate the pseudo resonant state in effective Green's function $\widetilde{\mathbf{G}}^{r / a, 0 / V}$ [Eqs. (37) and (38)], one has to calculate the self-energy by setting $\eta=0$ and resolve the retarded or advanced selfenergies with the aid of the group velocity $v_{k}=[\partial E(k) / \partial k] .{ }^{37}$

\section{CONCLUSION}

By orthogonalizing the Hamiltonian expressed in the nonorthogonal basis and considering the singularity of selfenergy $\Sigma^{r / a}\left(t, t^{\prime}\right)$ at $t=t^{\prime}$, we have generalized the solution [developed in Ref. 5] of the transient current driven either by a downward step voltage pulse or by a upward step pulse. This generalized result can be applied to both the quantum dot model and real molecular device. Based on the exact solution given in Ref. 5, we derived two approximate formulas that are suitable for numerical calculation of the transient current for molecular devices. We have tested our approximate formula in a quantum dot system where exact numerical solution exists. For the quantum dot system, we chose a Lorentzian linewidth (beyond wideband limit) and compared the time-dependent current calculated using both exact formula and our approximate formula. We found that for the opposite voltage $V_{L}(t)=-V_{R}(t)$, the results obtained from the exact formalism and two approximate scheme agree very well with each other especially in the downward pulse case. For the nonsymmetric voltage $V_{L}(t)=V(t), \quad V_{R}(t)=0$ or $V_{L}(t)=0, V_{R}(t)=-V(t)$, the second approximation is better.
This shows that our approximate formulas captured the essential physics of the transient current. In addition, it gives the correct initial current at $t=0$ and correct asymptotic current at $t \rightarrow \infty$. Since we have reduced the calculation from triple integral to single integral over the energy, the approximated approach reduces the computational cost drastically and it can be easily implemented in first-principles calculation for molecular devices. To demonstrate this, we calculated the transient current using the first approximated scheme with an opposite voltage $V_{L}(t)=-V_{R}(t)$ for two molecular structures: $\mathrm{Al}-\mathrm{C}_{5}-\mathrm{Al}$ and $\mathrm{Al}-\mathrm{C}_{60}-\mathrm{Al}$. Different from the quantum dot system, because of the complex electronic structure in molecular devices, transient currents oscillate rapidly in the first a few or tens femtoseconds as the bias voltage is switched, then gradually approach to the steadystate values. Furthermore, due to the resonant state in molecular devices, transient currents have a very long decay time $\tau$.

\section{ACKNOWLEDGMENTS}

This work was supported by a RGC under Grant No. HKU 705409P from the government of HKSAR.

\section{APPENDIX A: ORTHOGONALITY RELATION FOR THE NONORTHOGONAL BASIS}

For a system described by $H$, the time-independent eigenvalue equation is written as

$$
H|n\rangle=E_{n}|n\rangle .
$$

The eigenvectors $|n\rangle$ form an orthogonal complete basis set. However, in many systems such as a molecular device connected to external leads, the basis set constructed by eigenvectors is not convenient. We usually expand the eigenvector $|n\rangle$ in other basis $|\mu\rangle$, which is nonorthogonal complete set (or nearly complete).

$$
|n\rangle \simeq \sum_{\mu}|\mu\rangle\langle\mu \mid n\rangle
$$

the eigenvalue equation now becomes

$$
\begin{gathered}
\sum_{\mu} H|\mu\rangle\langle\mu \mid n\rangle=E_{n} \sum_{\mu}|\mu\rangle\langle\mu \mid n\rangle, \\
\sum_{\mu}\langle\nu|H| \mu\rangle\langle\mu \mid n\rangle=E_{n} \sum_{\mu}\langle\nu \mid \mu\rangle\langle\mu \mid n\rangle, \\
\sum_{\mu} \mathbf{H}_{\nu \mu} \Psi_{\mu}^{n}=E_{n} \sum_{\mu} \mathbf{S}_{\nu \mu} \Psi_{\mu}^{n},
\end{gathered}
$$

where $\mathbf{S}_{\nu \mu}=\langle\nu \mid \mu\rangle$. In the matrix form, we have $\mathbf{H} \Psi^{n}$ $=E_{n} \mathbf{S} \Psi^{n}$. If we use the self-energy to replace the effect of leads the effective Hamiltonian for the open system becomes $\mathbf{H}=\mathbf{H}_{0}+\boldsymbol{\Sigma}^{r}$. Since the effective Hamiltonian is not Hermitian, we can define the adjoint operator $\mathbf{H}^{\dagger}=\mathbf{H}=\mathbf{H}_{0}+\mathbf{\Sigma}^{a}$ and corresponding eigenequation becomes $\mathbf{H}^{\dagger}\left|\phi_{n}\right\rangle=E_{n}^{*} \mathbf{S}\left|\phi_{n}\right\rangle$. Then

$$
\boldsymbol{\Phi}^{m, \dagger} \mathbf{H} \boldsymbol{\Psi}^{n}=E_{n} \boldsymbol{\Phi}^{m, \dagger} \mathbf{S} \Psi^{n}
$$




$$
\boldsymbol{\Psi}^{n, \dagger} \mathbf{H}^{\dagger} \boldsymbol{\Phi}^{m}=E_{m}^{*} \boldsymbol{\Psi}^{n, \dagger} \mathbf{S}^{\dagger} \boldsymbol{\Phi}^{m} .
$$

Taking hermitian conjugate of Eq. (A5),

$$
\boldsymbol{\Phi}^{m, \dagger} \mathbf{H} \boldsymbol{\Psi}^{n}=E_{m} \boldsymbol{\Phi}^{m, \dagger} \mathbf{S} \boldsymbol{\Psi}^{n}
$$

From Eqs. (A4) and (A6), we have

$$
\boldsymbol{\Phi}^{n, \dagger} \mathbf{S} \boldsymbol{\Psi}^{m}=C_{m} \delta_{n m} .
$$

For the normalized wave function $\left|\psi_{n}\right\rangle$ and $\left|\phi_{n}\right\rangle$,

$$
\boldsymbol{\Phi}^{\dagger} \mathbf{S} \boldsymbol{\Psi}=\mathbf{I} .
$$

It is the usual orthogonality relation for eigenvectors expressed in a nonorthogonal basis set. For an hermitian Hamiltonian $\mathbf{H}=\mathbf{H}^{\dagger},\left|\psi_{n}\right\rangle=\left|\phi_{n}\right\rangle$, we have

$$
\boldsymbol{\Psi}^{\dagger} \mathbf{S} \boldsymbol{\Psi}=\mathbf{I} .
$$

\section{APPENDIX B: ORTHOGONALIZE HAMILTONIAN EXPRESSED IN NONORTHOGONAL BASIS}

In this appendix, we will show how to construct a new orthogonal basis from the atomic real-space nonorthogonal basis. We will transform the original Hamiltonian $\mathbf{H}$ which is expressed in the nonorthogonal basis into Hamiltonian $\widetilde{\mathbf{H}}$ expressed in the new orthogonal basis. Of course, instead of $\mathbf{S}$, the overlap matrix in the new basis will be $\mathbf{I}$.

Denoting nonorthogonal basis $|\mu\rangle$ and orthogonal basis $|j\rangle$, they are related by unitary transform operator $\mathcal{U}$

$$
\begin{gathered}
|\mu\rangle=\sum_{j}|j\rangle\langle j \mid \mu\rangle=\sum_{j}|j\rangle \mathcal{U}_{j \mu}, \\
\mathcal{U}_{j \mu}=\langle j \mid \mu\rangle,
\end{gathered}
$$

where we have used the completeness of orthogonal basis $|j\rangle$. Using the orthogonality $\langle i \mid j\rangle=\delta_{i j}$

$$
\sum_{\mu \nu}\langle i \mid \mu\rangle\langle\mu \mid \nu\rangle\langle\nu \mid j\rangle=\sum_{\mu \nu} \mathcal{U}_{i \mu} \mathbf{S}_{\mu \nu} \mathcal{H}_{\nu j}^{\dagger}=\delta_{i j},
$$

where we have used the completeness of nonorthogonal basis. In the matrix form, $\mathcal{U} \mathbf{S} \mathcal{U}^{\dagger}=\mathbf{I}$. We can formally define

$$
\mathcal{U}=\mathbf{S}^{-1 / 2}, \quad \mathcal{U}^{\dagger}=\left[\mathbf{S}^{-1 / 2}\right]^{\dagger} .
$$

Then new Hamiltonian $\tilde{\mathbf{H}}$ expressed in basis $|i\rangle$ can be expressed as

$$
\widetilde{\mathbf{H}}_{i j}=\langle i|H| j\rangle=\sum_{\mu \nu}\langle i \mid \mu\rangle\langle\mu|H| \nu\rangle\langle\nu \mid j\rangle=\sum_{\mu \nu} \mathcal{U}_{i \mu} \mathbf{H}_{\mu} \mathcal{U}_{\nu j}^{\dagger} .
$$

In the matrix form, $\widetilde{\mathbf{H}}=\mathbf{S}^{-1 / 2} \mathbf{H}\left[\mathbf{S}^{-1 / 2}\right]^{\dagger}$.
We now discuss how to find the matrix $\mathbf{S}^{-1 / 2}$. Without loss generality, we assume the real overlap matrix $\mathbf{S}$ satisfies eigenfunction $\mathbf{S V}=\mathbf{V} \operatorname{diag}\left(\lambda_{1}, \ldots, \lambda_{n}\right)$ with the eigenvalues $\lambda_{1}, \ldots, \lambda_{n}$ and eigenvectors $\mathbf{V}=\left[v_{1}, \ldots, v_{n}\right]$. Since $\mathbf{S}$ is real and symmetric, the eigenvectors are real and orthogonal, and it thus holds that $\mathbf{V}^{\dagger} \mathbf{V}=\mathbf{V} \mathbf{V}^{\dagger}=\mathbf{I}$. Then

$$
\begin{aligned}
\mathbf{S} & =\mathbf{V} \operatorname{diag}\left(\lambda_{1}, \ldots, \lambda_{n}\right) \mathbf{V}^{\dagger} \\
& =\mathbf{V} \operatorname{diag}\left(\sqrt{\lambda_{1}}, \ldots, \sqrt{\lambda_{n}}\right) \mathbf{V}^{\dagger} \mathbf{V} \operatorname{diag}\left(\sqrt{\lambda_{1}}, \ldots, \sqrt{\lambda_{n}}\right) \mathbf{V}^{\dagger} .
\end{aligned}
$$

It follows that

$$
\mathbf{S}^{1 / 2}=\mathbf{V} \operatorname{diag}\left(\sqrt{\lambda_{1}}, \ldots, \sqrt{\lambda_{n}}\right) \mathbf{V}^{\dagger} .
$$

From $\mathbf{S}^{-1 / 2} \mathbf{S}^{1 / 2}=\mathbf{I}$ and Eq. (B3), we have

$$
\begin{gathered}
\mathbf{S}^{-1 / 2} \mathbf{V} \operatorname{diag}\left(\sqrt{\lambda_{1}}, \ldots, \sqrt{\lambda_{n}}\right) \mathbf{V}^{\dagger}=\mathbf{I}, \\
\mathbf{S}^{-1 / 2} \mathbf{V} \operatorname{diag}\left(\sqrt{\lambda_{1}}, \ldots, \sqrt{\lambda_{n}}\right) \mathbf{V}^{\dagger} \mathbf{V} \operatorname{diag}\left(\frac{1}{\sqrt{\lambda_{1}}}, \ldots, \frac{1}{\sqrt{\lambda_{n}}}\right) \mathbf{V}^{\dagger} \\
=\mathbf{S}^{-1 / 2}=\mathbf{V} \operatorname{diag}\left(\frac{1}{\sqrt{\lambda_{1}}}, \ldots, \frac{1}{\sqrt{\lambda_{n}}}\right) \mathbf{V}^{\dagger} .
\end{gathered}
$$

In general, the dimension of matrix $\mathbf{S}$ is infinity, we cannot calculate its eigenvalue $\lambda_{i}$ and eigenvector $v_{i}$ by diagonalizing $\mathbf{S}$. However, in the tight-binding representation, the state $\mu$ and $\nu$ hardly overlap when their separation is large enough in real space, i.e., $\mathbf{S}_{\mu \nu} \approx 0$ for most of off-diagonal elements. Considering the periodic properties in semi-infinite leads, we can select a block matrix which is large enough to include all the overlap between leads and central molecular regions. For the nonorthogonal basis including several unit cell of atomic leads as a buffer layer into the central scattering region is enough to get a good screening for dc transport calculation. In transforming the Hamiltonian to the orthogonal basis needed for ac transport calculation, however, it turns out that we have to include at least 10 unit cells of atomic leads into the central scattering region. Partly because the overlap of orthogonal basis has longer range than that of nonorthogonal basis. With this large simulation box (finite dimension), we can calculate the overlap matrix $\mathbf{S}^{1 / 2}$ therefore transform $\mathbf{H}$ into $\tilde{\mathbf{H}}$. The accuracy of transformed Hamiltonian $\widetilde{\mathbf{H}}$ should be examined by comparing dc conductance obtained from the original Hamiltonian $\mathbf{H}$ and the transformed Hamiltonian $\widetilde{\mathbf{H}}$.

\footnotetext{
*jianwang@hkusua.hku.hk

${ }^{1}$ Molecular Electronics, edited by G. Cuniberti, G. Fagas, and K. Ricchter (Springer, Berlin, 2005).

${ }^{2}$ S. Kurth, G. Stefanucci, C.-O. Almbladh, A. Rubio, and E. K. U. Gross, Phys. Rev. B 72, 035308 (2005).
}

${ }^{3}$ Y. Zhu, J. Maciejko, T. Ji, H. Guo, and J. Wang, Phys. Rev. B 71, 075317 (2005).

${ }^{4}$ X. Qian, J. Li, X. Lin, and S. Yip, Phys. Rev. B 73, 035408 (2006).

${ }^{5}$ J. Maciejko, J. Wang, and H. Guo, Phys. Rev. B 74, 085324 
(2006).

${ }^{6}$ N. Sai, N. Bushong, R. Hatcher, and M. Di Ventra, Phys. Rev. B 75, 115410 (2007).

${ }^{7}$ X. Zheng, F. Wang, C. Y. Yam, Y. Mo, and G. H. Chen, Phys. Rev. B 75, 195127 (2007).

${ }^{8}$ G. Stefanucci, S. Kurth, A. Rubio, and E. K. U. Gross, Phys. Rev. B 77, 075339 (2008).

${ }^{9}$ J. Taylor, H. Guo, and J. Wang, Phys. Rev. B 63, 245407 (2001); 63, 121104 (2001)

${ }^{10}$ A. D. Stone, M. Ya. Azbel, and P. A. Lee, Phys. Rev. B 31, 1707 (1985).

${ }^{11}$ P. Johansson, Phys. Rev. B 41, 9892 (1990); T. Kwapiński, R. Taranko, and E. Taranko, ibid. 66, 035315 (2002); T. Kwapiński, ibid. 69, 153303 (2004).

${ }^{12}$ Q.-F. Sun and T.-H. Lin, J. Phys.: Condens. Matter 9, 3043 (1997); 9, 4875 (1997); Q.-F. Sun, B.-G. Wang, J. Wang, and T.-H. Lin, Phys. Rev. B 61, 4754 (2000); J. Q. You, C.-H. Lam, and H. Z. Zheng, ibid. 62, 1978 (2000); Z.-G. Zhu, G. Su, Q.-R. Zheng, and B. Jin, ibid. 68, 224413 (2003).

${ }^{13}$ S. Kohler, J. Lehmann, and P. Hnggi, Phys. Rep. 406, 379 (2005); L. E. F. Foa Torres and G. Cuniberti, C. R. Phys. 10, 297 (2009).

${ }^{14}$ M. Plihal, D. C. Langreth, and P. Nordlander, Phys. Rev. B 61, R13341 (2000).

${ }^{15}$ L. Y. Chen and C. S. Ting, Phys. Rev. B 41, 8533 (1990).

${ }^{16}$ W. R. Frensley, Phys. Rev. B 36, 1570 (1987).

${ }^{17}$ F. B. Anders and A. Schiller, Phys. Rev. Lett. 95, 196801 (2005).

${ }^{18}$ G. Stefanucci and C.-O. Almbladh, Europhys. Lett. 67, 14 (2004); Phys. Rev. B 69, 195318 (2004); N. Bushong, N. Sai, and M. Di Ventra, Nano Lett. 5, 2569 (2005).

${ }^{19}$ A. Schiller and S. Hershfield, Phys. Rev. B 62, R16271 (2000); Y. X. Xing, Q. F. Sun, and J. Wang, ibid. 75, 125308 (2007).
${ }^{20}$ By solving the time-dependent density matrix using the equation of motion method, Zheng et al.(Ref. 8) have calculated the transient current using the wideband limit.

${ }^{21}$ B. Wang, Y. Xing, L. Zhang, and J. Wang, Phys. Rev. B 81, 121103 (2010).

${ }^{22}$ N. S. Wingreen, A.-P. Jauho, and Y. Meir, Phys. Rev. B 48, 8487 (1993).

${ }^{23}$ A short account of this method can be found in Ref. 21.

${ }^{24}$ A.-P. Jauho, N. S. Wingreen, and Y. Meir, Phys. Rev. B 50, 5528 (1994).

${ }^{25}$ M. H. Pedersen and M. Büttiker, Phys. Rev. B 58, 12993 (1998)

${ }^{26}$ M. Buttiker, J. Phys.: Condens. Matter 5, 9361 (1993).

${ }^{27}$ Y. D. Wei and J. Wang, Phys. Rev. B 79, 195315 (2009).

${ }^{28}$ The exact solution of transient current on the orthogonal basis set has been derived in Ref. 5 .

${ }^{29}$ J. K. Viljas, J. C. Cuevas, F. Pauly, and M. Häfner, Phys. Rev. B 72, 245415 (2005).

${ }^{30}$ D. H. Lee and J. D. Joannopoulos, Phys. Rev. B 23, 4997 (1981); 23, 4988 (1981).

${ }^{31}$ M. Büttiker, A. Prêtre, and H. Thomas, Phys. Rev. Lett. 70, 4114 (1993).

${ }^{32}$ B. G. Wang, J. Wang, and H. Guo, Phys. Rev. Lett. 82, 398 (1999).

${ }^{33}$ A. Erdélyi, Asymptotic Expansions (Dover, New York, 1956).

${ }^{34}$ M. Brandbyge, J. L. Mozos, P. Ordejon, J. Taylor, and K. Stokbro, Phys. Rev. B 65, 165401 (2002).

${ }^{35}$ T. Markussen, M.S. thesis, Technical University of Denmark, 2006.

${ }^{36}$ B. Wang, Y. Xing, Y. Wei, L. Wan, and J. Wang, Carbon 43, 2786 (2005); B. Wang, Y. Xing, L. Wan, Y. Wei, and J. Wang, Phys. Rev. B 71, 233406 (2005).

${ }^{37}$ S. Sanvito, C. J. Lambert, J. H. Jefferson, and A. M. Bratkovsky, Phys. Rev. B 59, 11936 (1999). 